Mon. Not. R. Astron. Soc. 000, 1-?? (2012) Printed 6 February $2020 \quad$ (MN LATEX style file v2.2)

\title{
Characterization of M-stars in the LMC in the JWST era.
}

\author{
E. Marini, ${ }^{1,2 \star ~ F . ~ D e l l ' A g l i, ~}{ }^{2}$, M. Di Criscienzo ${ }^{2}$, D. A. García-Hernández ${ }^{3,4}$, \\ P. Ventura ${ }^{2}$, M. A. T. Groenewegen ${ }^{5}$, L. Mattsson ${ }^{6}$, D. Kamath ${ }^{7}$, S. Puccetti ${ }^{8}$, \\ M. Tailo ${ }^{9}$, and E. Villaver ${ }^{10}$ \\ ${ }^{1}$ Dipartimento di Matematica e Fisica, Universitá degli Studi Roma Tre, via della Vasca Navale 84, 00100, Roma \\ ${ }^{2}$ INAF, Osservatorio Astronomico di Roma, Via Frascati 33, 00077, Monte Porzio Catone, Italy \\ ${ }^{3}$ Instituto de Astrofísica de Canarias (IAC), E-38200 La Laguna, Tenerife, Spain \\ ${ }^{4}$ Departamento de Astrofísica, Universidad de La Laguna (ULL), E-38206 La Laguna, Tenerife, Spain \\ ${ }^{5}$ Koninklijke Sterrenwacht van België, Ringlaan 3, 1180 Brussels, Belgium \\ ${ }^{6}$ Nordita, KTH Royal Institute of Technology and Stockholm University, Roslagstullsbacken 23, SE-106 91 Stockholm, Sweden \\ ${ }^{7}$ Department of Physics and Astronomy, Macquarie University, Sydney, NSW 2109, Australia \\ ${ }^{8}$ ASI, Via del Politecnico, 00133 Roma, Italy \\ ${ }^{9}$ Dipartimento di Fisica e Astronomia 'Galileo Galilei', Univ. di Padova, Vicolo dell? Osservatorio 3, I-35122 Padova, Italy \\ ${ }^{10}$ Departamento de Fisica Teorica, Universidad Autonoma de Madrid, Cantoblanco 28049 Madrid, Spain
}

Accepted, Received; in original form

\begin{abstract}
We study the M-type asymptotic giant branch (AGB) population of the Large Magellanic Cloud (LMC) by characterizing the individual sources in terms of the main properties of the progenitors and of the dust present in the circumstellar envelope. To this aim we compare the combination of the spectroscopic and photometric data collected by Spitzer, complemented by additional photometric results available in the literature, with results from AGB modelling that include the description of dust formation in the wind. To allow the interpretation of a paucity stars likely evolving through the post-AGB phase, we extended the available evolutionary sequences to reach the $\mathrm{PN}$ phase. The main motivation of the present analysis is to prepare the future observations of the evolved stellar populations of Local Group galaxies that will be done by the James Webb Space Telescope (JWST), by identifying the combination of filters that will maximize the possibilities of characterizing the observed sources. The present results show that for the M-star case the best planes to be used for this purpose are the colour magnitude ([F770W]-[F2550W], [F770W]) and ( $\left.\mathrm{K}_{S^{-}}[\mathrm{F} 770 \mathrm{~W}],[\mathrm{F} 770 \mathrm{~W}]\right)$ planes. In these observational diagrams the sequences of low-mass stars evolving in the AGB phases before the achievement of the C-star stage and of massive AGBs experiencing hot bottom burning are clearly separated and peculiar sources, such as post-AGB, dual-dust chemistry and iron-dust stars can be easily identified.
\end{abstract}

Key words: galaxies: Magellanic Clouds - stars: AGB and post-AGB - stars: abundances - stars: AGB and post-AGB

\section{INTRODUCTION}

The stars evolving through the AGB provide an important feedback on their host system. During this phase they lose their entire external mantle, ejecting into the interstellar medium (ISM) large quantities of gas, partly contaminated by internal nucleosynthesis and mixing processes. Their circumstellar envelope is a favourable environment to the formation of dust (Woitke al. 1999), which is lost into their

\footnotetext{
* E-mail: ester.marini@uniroma3.it
}

surroundings, owing to the effects of the pulsation and of the radiation pressure acting on the dust grains. In this way, AGB stars participate to the dust cycle of the host galaxy (Javadi et al. 2016; Li et al. 2019).

Addressing these important topics is now possible thanks to the latest generation of models, that couple the simulation of the evolution of the central star and the description of the dust formation process, which, in turn, is coupled self-consistently with the dynamics of the wind (Ferrarotti \& Gail 2002, 2006). These preliminary investigations have proven extremely useful to foresee the amount 
of dust produced by stars of different mass and chemical composition, across the various stages of the AGB evolution (Dell'Agli et al. 2017; Di Criscienzo et al. 2013; Nanni et al. 2013, 2014; Ventura et al. 2012, 2014, 2018).

Despite these important progresses, the estimates of dust yields by AGB stars are still affected by several uncertainties, which can be broadly grouped into 3 distinct categories: a) the AGB evolution is strongly determined by the efficiency of two physical phenomena, still poorly known from first principles, namely convection and mass loss (Ventura \& D'Antona 2005a,b; Karakas \& Lattanzio 2014); b) the dynamics of the wind is characterized by the formation of shocks, which provoke significant deviations with respect to the isotropic, stationary schematization currently used (Bowen 1988; Cherchneff 2006); c) Dust production mechanism itself is still affected by several uncertainties, related to the scarce knowledge of the sticking coefficients of the molecules on the solid particles and to the formation entalpies of some solid compounds (Ferrarotti \& Gail 2006; Gail et al. 2013).

On the observational ground, photometry and spectroscopy in the infrared (IR) domain prove valuable tools to improve the understanding of the evolution and of the dust production mechanism by AGB stars. Indeed, when dust is formed a significant fraction of the overall energy released is emitted in the IR spectral region. Furthermore, the IR spectrum is characterized by various features, each associated to a specific dust species; this is important to deduce the mineralogy of the dust formed.

The LMC has been so far the best laboratory to test AGB evolution theories. This is due to its relative proximity ( $\sim 50 \mathrm{Kpc}$, Feast 1999) and low average reddening $(E(B-V) \sim 0.075$, Schlegel et al. 1998), which allowed the observation of the evolved stellar population by means of several surveys. The most recent and complete exploration has been achieved via the Surveying the Agents of a Galaxy's Evolution Survey (SAGE), with the Spitzer Space Telescope (Meixner et al. 2006), that provided IR data taken with the InfraRed Array Camera (IRAC, with filters centered at 3.6, 4.5, 5.8 and $8.0 \mu \mathrm{m}$ ) and the Multi-band Imaging Photometer (MIPS, with filter centered at $24 \mu \mathrm{m}$ ) of $\sim 6.5$ million sources, $\sim 17000$ out of which were classified as AGB stars by Riebel et al. (2010).

The availability of this robust body of observational data has allowed the study of the dust enrichment from stellar sources from two different perspectives. Several authors used synthetic spectra, obtained by varying the parameters of the central object and the dust composition, to reproduce the position of the observed sources in the observational planes built with the IRAC and MIPS filters (Srinivasan et al. 2009, 2010, 2011; Riebel et al. 2012). A different and complementary approach was followed by Dell'Agli et al. (2014a, 2015a), who used stellar evolutionary tracks to characterize the individual sources, in terms of mass, chemical composition and formation epoch of the progenitors, and of the amount and mineralogy of the dust in the circumstellar envelope. Nanni et al. (2019) used a similar analysis to derive an estimate of the overall dust production rate by evolved stars in the Magellanic Clouds (hereinafter MC).

A further step towards the interpretation of the IR observations of LMC stars is possible via the analysis of spectroscopic data taken with the Spitzer's Infrared Spectro- graph (IRS), which provided detailed mid-IR spectral distribution of more than 1000 point sources in the LMC. The fit of the IR spectra allows a wider and deeper exploration of the various factors affecting the spectral energy distribution, in comparison to the analysis based on the different magnitudes. This approach was followed by Jones et al. (2014) and Groenewegen \& Sloan (2018, GS18) to characterize oxygenrich AGB stars.

In the near future, the studies aimed at understanding how dust production in the envelope of AGB stars works will receive a robust push, with the launch of the $J W S T$, that will revolutionize our understanding of the evolved stellar populations in the local Universe. The large aperture $(6.5 \mathrm{~m})$ and the subarsecond spatial resolution will allow the study of resolved dusty stellar populations at moderate and large distances, up to $\sim 4 \mathrm{Mpc}$ (Jones et al. 2017). The Mid-Infrared Instrument (MIRI; Rieke et al. 2015), mounted onboard the $J W S T$, will provide spectroscopy in the $5-28.5 \mu \mathrm{m}$ range (Bouchet et al. 2015), thus providing a unique opportunity to study the evolution of AGB stars and the dust formation process in their expanding wind, in a large variety of environments. Combination of near-IR and mid-IR data to study the evolved stellar population has been so far successfully applied to the MC. By analyzing the results from the DUSTiNGS survey, Dell'Agli et al. (2016, 2018b, 2019) attempted a similar approach to study AGB stars in the Local Group galaxies IC1613, IC10 and Sextans A; however, these studies, based on photometry results limited to wavelengths below $\sim 5 \mu \mathrm{m}$, could not benefit of the information of the long-wavelength domain of the mid-IR flux. The $J W S T$ data will allow the application of this methodology to all the galaxies of the Local Group and possibly beyond.

Against this background, we have started a research project with the goal of providing a thorough interpretation of the IR data of the stars in the LMC that exhibit an IR excess. In this paper we restrict our attention to the sample of oxygen-rich stars, while we will address carbon stars in a forthcoming work. In the wake of the analysis done in Jones et al. (2014), we will combine results from IRAC and MIPS photometry with IRS data, in the attempt of giving an exhaustive characterization of the individual sources, in terms of the main properties of their progenitors, of the specific AGB stage they are evolving through and of the properties of the dust in their surroundings. To consider the whole spectral distribution, we will also take into account optical and near IR photometric data, when available. This study represents a step forward with respect to the works by Dell'Agli et al. (2014a, 2015a), which were based on photometric data only. The method followed here is different and complementary from Jones et al. (2014), as it is based on results from AGB evolution and dust formation modelling.

Because the present work is projected into a $J W S T$ perspective, similarly to Jones et al. (2017) we will consider observational planes obtained by the combination of MIRI filters. Our goal is to select the observational planes that must be used in order to obtain the most exhaustive characterization of the individual sources observed, in terms of chemical composition, mass and formation epoch of the progenitors, of the degree of obscuration of the stars and of the mineralogy of the dust present in the circumstellar envelope. A detailed care will be devoted to understand the planes where the different classes of the sources observed can be easily dis- 
tinguished and where the obscuration trends for oxygen-rich stars are most clearly defined. This step is crucial to set up a methodology that will be extended to the galaxies in the Local Group, once the JWST data will become available.

\section{AGB AND DUST FORMATION MODELLING}

The discussion presented in the following sections will be based on AGB evolution models which include the description of dust formation in the circumstellar envelope. The evolutionary sequences on which the present work is based are the same ones used in a series of papers published by our group to characterize the evolved stellar populations of the Magellanic Clouds (Dell'Agli et al. 2014b, 2015a,b) and Local Group galaxies (Dell'Agli et al. 2016, 2018b, 2019). The interested reader can find in these papers and in the recent review by Karakas \& Lattanzio (2014) a thorough discussion of the AGB evolution of $1-8 M_{\odot}$ stars. Here we briefly recall the most relevant properties.

\subsection{The physical and chemical evolution of AGB stars}

The left and middle panels of Fig. 1 show the evolution of the luminosity and of the ${ }^{12} \mathrm{C}$ surface mass fraction of stars of different mass. The grey parts of the tracks correspond to the C-star phase. Among the various chemical species we focus on ${ }^{12} \mathrm{C}$, because surface carbon is extremely sensitive to the efficiency of both hot bottom burning (hereinafter HBB, Renzini \& Voli 1981) and third dredge-up (TDU, Iben 1974). The models presented in Fig. 1 have $Z=8 \times 10^{-3}$, the metallicity shared by the majority of the stars in the LMC, now evolving through the AGB phase (Harris \& Zaritsky 2009). The current mass of the star is shown on the abscissa.

HBB consists in the activation of a series of p-capture reactions at the base of the convective envelope, which modifies the relative distribution of the various chemical species. The ignition of this process requires temperature at the bottom of the surface convective zone of the order of $\sim 30 \mathrm{MK}$, a condition that requires core masses $\sim 0.8 M_{\odot}$ (Ventura et al. 2013), reached only by stars of initial mass above $3.5 M_{\odot}{ }^{1}$.

The ignition of HBB (see the tracks of 4, 5,6 $M_{\odot}$ models in Fig. 1) leads to a fast rise in the luminosity of the star (Blöcker \& Schönberner 1991), which increases during the first part of the AGB evolution, until reaching a peak of the order of $5 \times 10^{4}-10^{5} L_{\odot}$, which is higher the larger the initial mass of the star is; in the final AGB phases the luminosity diminishes, because the general cooling of the external regions weakens (and eventually turns off) HBB (Mazzitelli et al. 1999).

The activation of HBB favours the destruction of the surface carbon, exposed to proton fusion at the base of the envelope; this effect can be seen in the steep drop (by a factor $\sim 20$ ) in the surface ${ }^{12} \mathrm{C}$, clearly visible in middle panel of

1 The minimum initial mass required to activate $\mathrm{HBB}$ during the AGB evolution is indeed dependent on the metallicity of the star. It is $3.5 M_{\odot}$ for the $Z=8 \times 10^{-3}$ chemistry used here, whereas for metal-poor stars with $Z \leqslant 10^{-3}$ it is $\sim 3 M_{\odot}$.
Fig. 1. Depletion of surface carbon requires temperatures at the base of the envelope of the order of $\sim 40 \mathrm{MK}$, which are reached by all the stars experiencing $\mathrm{HBB}$, independently of the chemical composition. Other nuclear reactions, e.g. proton captures by oxygen nuclei and the activation of the $\mathrm{Ne}-\mathrm{Na}$ and $\mathrm{Mg}-\mathrm{Al}-\mathrm{Si}$ nucleosynthesis, demand temperatures close to $100 \mathrm{MK}$, that are reached only by AGB stars of low metallicity ( $Z \leqslant 10^{-3}$, Dell'Agli et al. 2018a). The tracks corresponding to the $1.25,1.5,2.5 M_{\odot}$ models in Fig. 1 represent low-mass AGB stars, not experiencing $\mathrm{HBB}$. In these stars the luminosity rises during the AGB phase, owing to the increase in core mass, until reaching a final value, slightly below $\sim 10^{4} L_{\odot}$ (Boothroyd \& Sackmann 1988).

The only mechanism able to alter the surface chemical composition of these stars is TDU, which provokes a gradual increase in the surface carbon, evident in the middle panel of Fig. 1. Repeated TDU events lead to the formation of carbon stars, with a surface $\mathrm{C} / \mathrm{O}$ ratio above unity. This condition is reached by stars with initial mass $1<M / M_{\odot}<3^{2}$. The largest values of surface carbon mass fraction, slightly above $\sim 1 \%$, are reached by the stars of initial mass $\sim 2.5-3 M_{\odot}$, that are exposed to a higher number of TDU episodes before they loose the external envelope, compared to their lower mass counterparts (Karakas 2010; Karakas et al. 2018).

On the physical side, the enrichment in carbon content in the envelope determines a significant increase in the surface opacities (Marigo 2002), which favours the expansion of the external regions of the star and the increase in the mass loss rate, that exceeds $\sim 10^{-5} M_{\odot} /$ yr $($ Ventura \& Marigo 2009, 2010). The stars of initial mass below $2 M_{\odot}$ become C-stars after a significant fraction of the envelope was lost during the previous AGB phases; for the reasons given above the mass loss rates experienced after the $\mathrm{C}$-star stage is reached are so large that the residual envelope is lost before further thermal pulses (TPs) are experienced.

A general behaviour of AGB stars is that the loss of the external mantle is accompanied by the cooling of the external regions, which makes the stars readjust on a more and more expanded configuration (Vassiliadis \& Wood 1993). As TP-AGB stars are pulsating stars, this reflects into a gradual increase of the pulsation period $(\mathrm{P})$ of the star. Massive AGB stars are expected to reach extremely long periods, in the range 1000-2000d, during the peak of the HBB activity; in the finale phases, after most of the envelope is lost and the star starts to contract, the pulsation periods decrease.

\subsection{Dust production in AGB stars}

In the schematization adopted here dust formation occurs while the gas is expanding away from the central star, in the form of wind. The key-factor affecting the mineralogy of the dust formed is the $\mathrm{C} / \mathrm{O}$ ratio, owing to the high stability of the CO molecule (Sharp \& Huebner 1990). In oxygen-rich stars the formation of silicates, alumina dust and solid iron occurs, whereas in the envelope of carbon stars the main

2 The lower limit in mass is slightly dependent on metallicity. Indeed in metal-poor stars the achievement of the C-star condition is easier, because the lower oxygen; therefore, the lower mass threshold required to reach the C-star phase during the AGB evolution is smaller. 

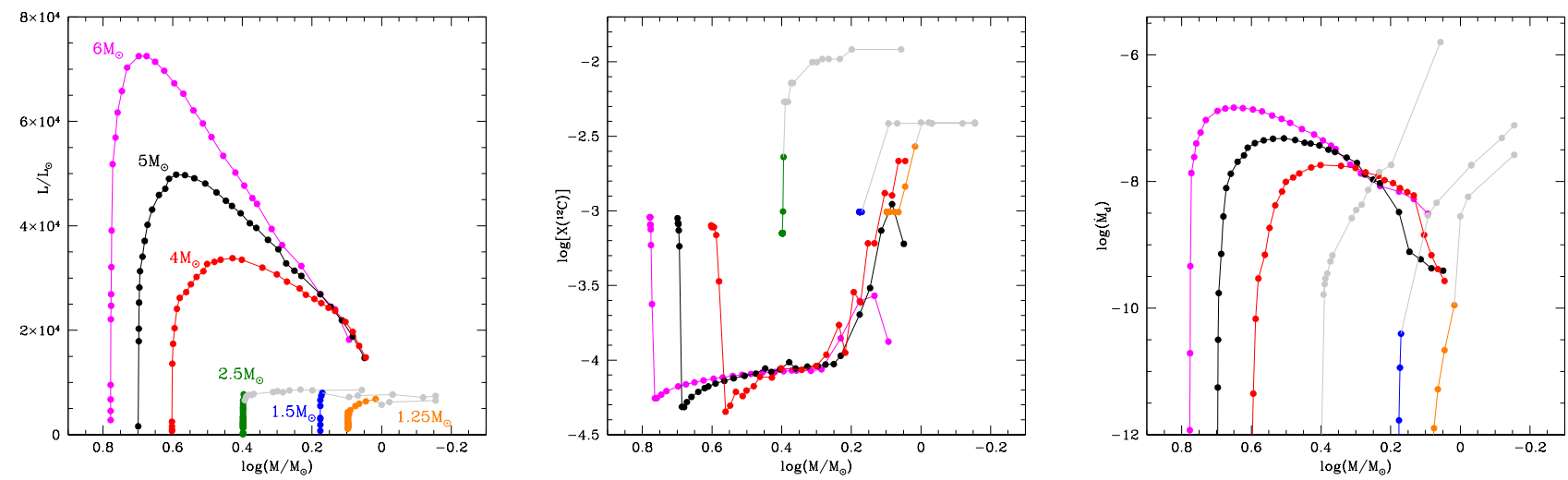

Figure 1. The variation during the AGB phase of the luminosity (left panel), surface carbon mass fraction (middle) and dust production rate (right), as a function of the current mass of the star (shown in a logarithmic scale), of stars of different mass and metallicity $Z=8 \times 10^{-3}$. The color-coding is as follows: orange points - $1.25 M_{\odot}$; blue - $1.5 M_{\odot}$; green - $2.5 M_{\odot} ;$ red - $4 M_{\odot} ;$ black - $5 M_{\odot}$; magenta $-6 M_{\odot}$; The points shown in grey refer to the carbon star phases.

dust species formed are solid carbon, $\mathrm{SiC}$ and solid iron (Ferrarotti \& Gail 2006).

The calculation of the extinction coefficients, required to determine the acceleration of the wind, is based on the following set of optical constants: silicates (Ossenkopf et al. 1992); alumina dust (Begemann, et al. 1997); iron (Ordal et al. 1988), solid carbon (Hanner 1988); silicon carbide (Pegourie 1988).

The dust mass loss rates $\left(\dot{M}_{d}\right)$ for the AGB models discussed so far are shown in the right panel of Fig. 1. These results are thoroughly discussed in Dell'Agli et al. (2015a). The phases with the largest $\dot{M}_{d}$ are those showing up the highest degree of obscuration, thus the largest IR excess.

Massive AGB stars never reach the C-star stage, thus they do not produce any carbon dust. The $\dot{M}_{d}$ of these objects is mostly determined by the values of the gas mass loss rate, which reflects the run of the luminosity ${ }^{3}$. This behaviour is clear when comparing the evolution of luminosity and dust mass loss rate in $M \geqslant 4 M_{\odot}$ stars, shown in the left and right panels of Fig. 1.

In low-mass stars the mineralogy of the dust formed consists in silicates, alumina dust and iron, during the first part of the AGB evolution, then changes to carbonaceous species, when they become carbon stars. As shown in Fig. 1, the achievement of the C-star stage provokes a significant increase in $\dot{M}_{d}$.

We now focus on the dust produced during the O-rich phase, which is most relevant to the present work. Most of the dust from M-stars is produced by $M \geqslant 4 M_{\odot}$ stars, during the phases that follow the ignition of HBB. Inspection of the right panel of Fig. 1 reveals that these stars experience an initial AGB phase, with poor dust production, followed by phases characterized by the presence of large quantities of dust in the circumstellar envelope, with $\dot{M}_{d} \sim 10^{-8}-10^{-7} M_{\odot} /$ yr. Low-mass objects evolve as M-

3 The increase in the mass loss rate with the luminosity, which is general, is particularly steep in the present models, that adopt the treatment of mass loss by Blöcker \& Schönberner (1991). stars during the initial part of the AGB phase, before they turn to C-stars. The dust produced by these objects during the O-rich phase is negligible, because their mass loss rate, below $10^{-6} M_{\odot} / \mathrm{yr}$, is too small to allow formation of dust in meaningful quantities. An exception to this is represented by low-mass stars of initial mass around $1-1.5 M_{\odot}$. These stars reach the C-star phase only in the very final AGB stages, when a significant fraction of the envelope is lost. During the end of the O-rich phase, before turning to C-stars, they evolve at cool effective temperatures and their mass loss rate reaches $\sim 10^{-6} M_{\odot} /$ yr: these conditions favour the formation of small, but not negligible, amount of silicates and alumina dust.

\subsection{The evolution of the spectral energy distribution of M-type AGB stars}

The results from stellar evolution modelling coupled with the description of dust formation in the wind allows the determination of the change in the spectral energy distribution (SED) of the star during the AGB phase. To this aim, we selected some representative points along the individual sequences (tipically $\sim 20$ between two successive TPs) and used the code DUSTY (Nenkova et al. 1999) to calculate the shape of the expected emission. The input needed to this computation are the temperature of the condensation region, luminosity, effective temperature of the star, the size of the dust grains formed and the optical depth, which in our case is assumed at the wavelength $\lambda=10 \mu \mathrm{m}$.

From the discussion in the previous section we know that in the context of $\mathrm{M}$ stars significant dust quantities are produced by $M \leqslant 2 M_{\odot}$ stars, in the phases previous to the achievement of the C-star stage, and by massive AGB stars, after the beginning of HBB. We discuss these two cases below, separately.

The former evolve as $\mathrm{M}$ stars for most of their AGB life, until they become carbon stars. $\dot{M}_{d}$ becomes higher and higher during the AGB phase (see the blue and magenta tracks, corresponding to the $1.5 M_{\odot}$ and $1.25 M_{\odot}$ models, in the right panel of Fig. 1), because the gradual 

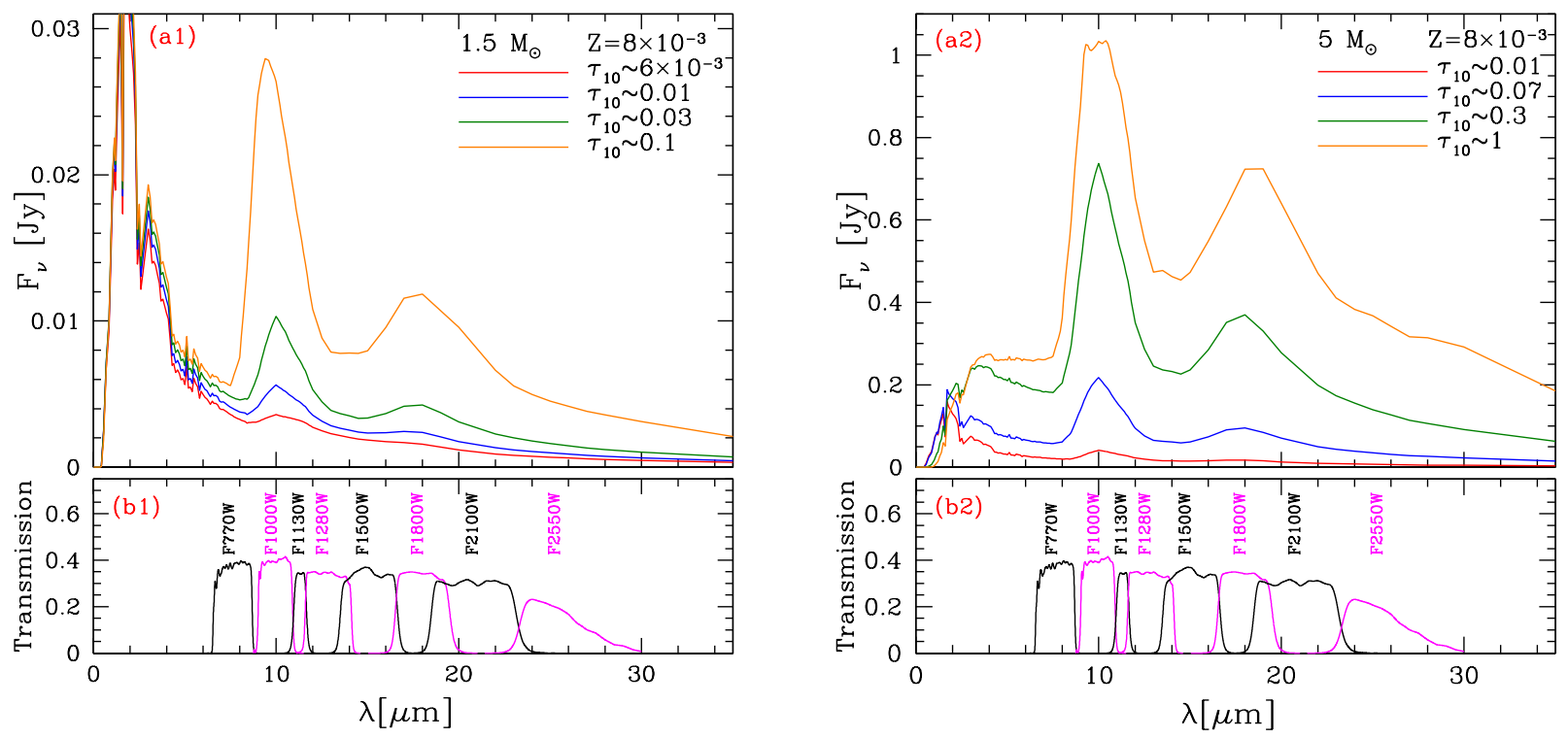

Figure 2. The expected variation of the SED during 4 evolutionary phases of the AGB evolution of a star of initial mass $1.5 M_{\odot}$ (a1) and $5 M_{\odot}(\mathrm{a} 2)$. The 4 phases are characterized by different degrees of obscuration of the circumstellar envelope, here represented by the values of $\tau_{10}$, indicated in the two panels. Fluxes are calibrated to the distance of the LMC (Feast 1999). Panels b1 and b2 show the transmission curves of MIRI filters in el. $\mathrm{sec}^{-1}$.

loss of the envelope and the general cooling of the external regions favour the increase in the mass loss rate. The degree of obscuration in the SED of these objects thus increases during the M-stage. This is shown in panel a1 of Fig. 2, that shows the expected evolution of the SED of a star of initial mass $1.5 M_{\odot}$; the different lines correspond to four evolutionary stages, from the initial AGB phases (red line), when dust formation is inefficient, to the phases immediately before the achievement of the C-star phase (orange track), when the optical depth grows to $\tau_{10} \sim 0.1$. The increase in the amount of dust formed makes the SED of the star to exhibit two prominent features, at $9.7 \mu \mathrm{m}$ and $18.7 \mu \mathrm{m}$, associated to the presence of silicates. Furthermore, the SED in the whole mid-IR region of the spectrum is lifted.

The stars that reach the highest $\dot{M}_{d}$ during the M-stage are those that experience HBB (see red, black and magenta lines in the right panel of Fig. 1), the progeny of $M \geqslant 4 M_{\odot}$ stars. As shown in Fig. $1, \dot{M}_{d}$ of this class of objects increases during the first part of the AGB evolution, until the maximum luminosity and mass loss rate is reached, then decreases when the efficiency of HBB starts to diminish. According to our modelling the largest optical depths attained are of the order of $\tau_{10} \sim 1$ (Dell'Agli et al. 2015a).

Panel a2 of Fig. 2 shows the expected evolution of the SED of a $5 M_{\odot}$ star, from the beginning of the AGB phase (red line), until the point of highest obscuration, reached in conjunction with the largest luminosity (orange).

The changes in the SED of these two classes of objects allow the determination of the general obscuration patterns traced by these stars in the different observational planes, that are useful to characterize the evolved $M$ stars in the LMC and in other galaxies.

\subsection{Post-AGB evolution modelling}

A few sources belonging to the sample published by Jones et al. (2012) show the presence of cool dust in the circumstellar envelope, suggesting that dust production has stopped and that the dust currently observed was produced during earlier evolutionary phases. The SED of these stars present a peak in the optical that rules out the possibility that they are AGB stars. Based on these factors, Marini et al. (2019b) suggested that these stars have left the AGB and are evolving through the post-AGB phase.

To further investigate and interpret these objects, we extended the evolutionary computations, so far limited to the AGB stage ${ }^{4}$, to the post-AGB phase. These sources, investigated by Marini et al. (2019b) and further discussed in detail in Sect. 4.5, have luminosities below $10^{4} L_{\odot}$, compatible with the evolution of stars of initial masses below $2 M_{\odot}$. Therefore, we decided to focus on the post-AGB phase of $M \leqslant 2 M_{\odot}$ stars, with metallicity $Z=8 \times 10^{-3}$.

The present post-AGB computations were selfconsistently resumed from the $Z=8 \times 10^{-3}$ AGB models used by Dell'Agli et al. (2015a). For the stars not reaching the C-star stage we modeled mass loss according to eq. 6 in Miller Bertolami (2016). For C-stars we chose, somewhat arbitrarily, to keep the description by Wachter et al. (2002, 2008). This choice will likely affect the timescale of the postAGB evolution, but is not relevant for the determination of the excursion of the evolutionary track on the HR diagram.

The main properties of the post-AGB models are reported in Table. 1. As discussed by Miller Bertolami (2016), the definition of the start of the post-AGB phase is not

\footnotetext{
4 When a few tenths of solar masses were left in the envelope.
} 
Table 1. Main physical and chemical properties of the post-AGB models discussed in the text. Cols. 1-3 indicate the initial mass of the star, the mass at the beginning of the AGB phase and the final mass. The time scales reported in col. 4 and 5 indicate, respectively, the age of the star and the crossing time, defined as the time interval from the point when the mass of the envelope drops below $1 \%$ of the stellar mass and the time when the effective temperature becomes $\log T_{\text {eff }}=3.85$. The last four cols. report the surface chemical composition, in terms of the mass fractions of helium, carbon, nitrogen and oxygen.

\begin{tabular}{ccccccccc}
\hline$M / M_{\odot}$ & $M_{A G B} / M_{\odot}$ & $M_{f} / M_{\odot}$ & $\tau_{e v}(\mathrm{Gyr})$ & $\tau_{t r}(\mathrm{Kyr})$ & $\mathrm{X}(\mathrm{He})$ & $\mathrm{X}(\mathrm{C})$ & $\mathrm{X}(\mathrm{N})$ & $\mathrm{X}(\mathrm{O})$ \\
\hline 1.00 & 0.75 & 0.552 & 9.23 & 3.8 & 0.281 & $8.86 \mathrm{e}-4$ & $4.72 \mathrm{e}-4$ & $4.16 \mathrm{e}-3$ \\
1.10 & 0.90 & 0.575 & 6.52 & 8.8 & 0.281 & $9.74 \mathrm{e}-4$ & $3.08 \mathrm{e}-4$ & $4.24 \mathrm{e}-3$ \\
1.25 & 1.10 & 0.596 & 4.22 & 3.3 & 0.290 & $8.59 \mathrm{e}-4$ & $3.24 \mathrm{e}-4$ & $4.66 \mathrm{e}-3$ \\
1.40 & 1.25 & 0.590 & 2.91 & 4.2 & 0.284 & $3.91 \mathrm{e}-3$ & $3.02 \mathrm{e}-4$ & $4.39 \mathrm{e}-3$ \\
1.60 & 1.50 & 0.602 & 1.89 & 3.4 & 0.284 & $3.86 \mathrm{e}-3$ & $2.99 \mathrm{e}-4$ & $4.40 \mathrm{e}-3$ \\
1.75 & 1.75 & 0.622 & 1.42 & 2.9 & 0.285 & $4.84 \mathrm{e}-3$ & $3.14 \mathrm{e}-4$ & $4.62 \mathrm{e}-3$ \\
2.00 & 2.00 & 0.617 & 0.97 & 3.0 & 0.286 & $6.21 \mathrm{e}-3$ & $2.97 \mathrm{e}-4$ & $4.68 \mathrm{e}-3$ \\
\hline
\end{tabular}

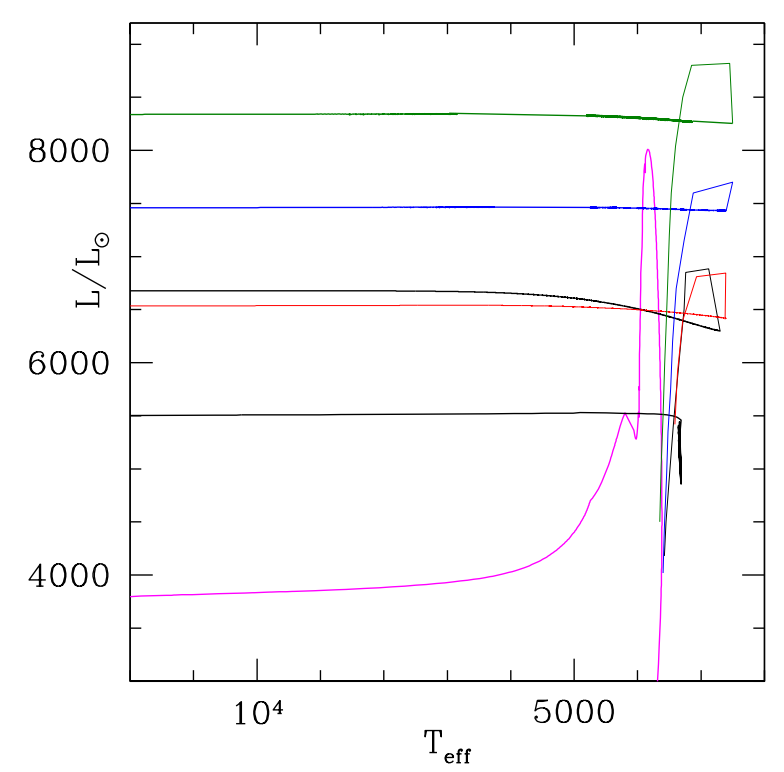

Figure 3. The path traced in the HR diagram by stars of initial mass $1 M_{\odot}$ (magenta line), $1.1 M_{\odot}$ (black), $1.25 M_{\odot}$ (red), $1.4 M_{\odot}$ (black), $1.6 M_{\odot}$ (blue), $1.75 M_{\odot}$ (green), during the latest AGB phases and the post-AGB phase.

rigorous, considering that the behaviour of the stars, particularly the beginning of the excursion of the evolutionary track to the blue, is dependent on the core mass. We assume that the AGB evolution ends when the mass of the envelope drops below $1 \%$ of the mass of the star and chose the point when the effective temperature is $\log T_{\text {eff }}=3.85$ as the intermediate stage between the post-AGB phase and the PN evolution. The time interval between these two evolutionary stages, proposed by Miller Bertolami (2016) as indicator of the transition time scale from the AGB to the post-AGB phase, is reported on column 5 of Table 1.

The path traced by the evolutionary tracks in the HR diagram of the models reported in Table 1 are shown in Fig. 3. Since the sources that we want to discuss here have $T_{\text {eff }}<10^{4} \mathrm{~K}$, we restrict our attention to effective temperatures below $1.2 \times 10^{4} \mathrm{~K}$. For each mass we show a few points regarding the very final AGB phases and the whole post-AGB evolution.

These results will be used later to discuss a few stars in the sample that are most likely evolving through the postAGB phase.

\section{THE LMC SAMPLE}

In this paper we use the LMC sample of 71 O-rich AGB spectra $^{5}$, observed using Spitzer IRS (Jones et al. 2012) and classified according to the decision-tree scheme proposed by Woods et al. (2011). The sample includes point sources from the SAGE-Spec legacy survey of the LMC (Kemper et al. 2010), a spectroscopic follow-up to the SAGE-LMC project (Meixner et al. 2006) and has been supplemented from archival Spitzer IRS observation within the SAGELMC footprint (Woods et al. 2011). The detailed description of the original target selection, the observing strategy and the techniques used in the data reduction for the SAGE-Spec legacy programme are discussed in Kemper et al. (2010). To study the distribution on the observational planes built from MIRI filters, we use the mid-IR magnitudes ([F770W], [F1000W], [F1130W], [F1280W], [F1500W], [F1800W], [F2100W], [F2550W]) calculated by Jones et al. (2017), who integrated the Spitzer IRS spectra of each source over the MIRI spectral response (see panels b1 and b2 in Fig. 2). The fluxes for the F560W filter are not available since the Spitzer IRS spectra cover the wavelength range $5.3-8 \mu \mathrm{m}$. For all the sources the associated broadband photometry, including optical $U B V I$ photometry from the Magellanic Clouds Photometric Survey (Zaritsky et al. 2004), Two Micron All Sky Survey (2MASS) $J H K_{S}$ photometry (Skrutskie et al. 2006), mid-IR photometry from Infrared Array Camera (IRAC 3.6, 4.5, 5.8, $8.0 \mu \mathrm{m}$ ) and Multi-Band Imaging Photometer for Spitzer (MIPS $24 \mu \mathrm{m}$ ), was compiled from the SAGE catalogue (Meixner et al. 2006). For 23 of these objects there is no spectral coverage by the Spitzer-IRS at $\lambda>14.2 \mu \mathrm{m}$, so mid-IR photometry beyond this wavelength is not available. The list of the stars analyzed in this work is reported in Table 2. When available, in column 3 we report the primary periods obtained by the OGLE collaboration (Soszyński, et

\footnotetext{
${ }^{5}$ http://irsa.ipac.caltech.edu/data/SPITZER/SAGE
} 
al. 2009), that phases well the temporal sequence of observations.

\section{M-TYPE AGB STARS: A JWST PERSPECTIVE}

Fig. 4 shows the distributions of the stars studied by Jones et al. $(2012,2014,2017)$ in four colour-magnitude planes obtained by means of the fluxes in some of the JWST filters. Three out of the four planes, built with the MIRI filters, were proposed by Jones et al. (2017) to distinguish different classes of objects and identify C- and M-type AGB stars. In addition, we discuss the $\left(K_{S}-[\mathrm{F} 770 \mathrm{~W}],[\mathrm{F} 770 \mathrm{~W}]\right)^{6}$ plane to explore the possible use of the combination of near-IR and mid-IR photometry to deduce the properties of the stars observed.

Our approach is the following. We first characterize the individual sources based on the comparison between their position in the afore mentioned planes and the path traced by the evolutionary tracks, calculated by convolving the synthetic SEDs, discussed in Sect. 2.3, with the transmission curves of the MIRI filters, shown in panels b1 and b2 of Fig. 2, and of 2MASS $\mathrm{K}_{S}$ filter. On this regard, we believe important to underline that low-mass stars and massive AGBs define two distinct obscuration patterns, where the colors and magnitudes are primarily determined by the luminosity and the degree of obscuration, i.e. the optical depth. We will return to this point in Sect. 4.2 and 4.3. We further define the details of the dust mineralogy by the tight comparison between the IRS spectra and the synthetic SEDs, corresponding to the individual points along the tracks.

Fig. 5 shows the distribution in the Period - Luminosity (PL) diagram of the stars in our sample for which OGLE periods are available. In some cases this plane will help us to better characterize the sources.

In the following we discuss different groups of stars, separated according to their degree of obscuration and/or peculiar features present in the SED, in turn connected with the mineralogy of the dust in the circumstellar envelope.

\subsection{Scarcely obscured, no-dusty stars}

The stars indicated with orange pentagons in Fig. 4 show no trace of dust in their surroundings. The SED of these objects can be safely reproduced by assuming optical depths close to zero, indicating a negligible degree of obscuration. Based on the luminosities obtained by SED fitting, we deduce that this group of sources is mainly composed by lowmass stars, that have not reached the C-star stage. In the ([F770W]-[F2550W], [F770W]) plane they populate the region within $0<[\mathrm{F} 770 \mathrm{~W}]-[\mathrm{F} 2550 \mathrm{~W}]<0.7$, separated from the other sources.

In the $([\mathrm{F} 1000 \mathrm{~W}]-[\mathrm{F} 1500 \mathrm{~W}],[\mathrm{F} 1000 \mathrm{~W}])$ and $([\mathrm{F} 1000 \mathrm{~W}]-$ $[\mathrm{F} 2100 \mathrm{~W}],[\mathrm{F} 1000 \mathrm{~W}])$ planes these stars can be identified by means of the low $[\mathrm{F} 1000 \mathrm{~W}]$ fluxes, consistent with the scarce presence of silicates in the circumstellar envelope, that prevents the formation of the $9.7 \mu \mathrm{m}$ feature.

6 We expect to obtain similar trends when using the NIRCam F210M filter.
In the $\left(K_{S}-[\mathrm{F} 770 \mathrm{~W}],[\mathrm{F} 770 \mathrm{~W}]\right)$ plane their identification is more tricky, because they mix with obscured stars with optical depths $0.005<\tau_{10}<0.1$. This is because when $0.005<\tau_{10}<0.1$ : a) the formation of silicates increases the flux in the spectral region around $10 \mu \mathrm{m}$, provoking at the same time a dip around $\sim 8 \mu \mathrm{m}$, that decreases the [F770W] flux (see e.g. the blue line in the right panel of Fig. 2); b) there is no significant shift of the SED towards mid-IR wavelengths, thus the $K_{S}$ flux is only scarcely affected by dust reprocessing. Therefore, the stars with a small degree of obscuration are characterized by $K_{S^{-}}[\mathrm{F} 770 \mathrm{~W}]$ colours and $[\mathrm{F} 770 \mathrm{~W}]$ magnitudes not significantly different from those exhibited by the unobscured stars discussed here.

In the planes shown in the top panels of Fig. 4 the stars with no dust span a $[\mathrm{F} 770 \mathrm{~W}]$ range of approximately 2 mag. This reflects the different luminosities, which we find (by SED fitting) to be in the range $5 \times 10^{3}-3 \times 10^{4} L_{\odot}$, consistent with the heterogeneity in the mass and formation epoch of the progenitors of these sources. This same analysis cannot be done in the planes shown in the bottom of the figure, because the lack of the Spitzer IRS data at wavelengths $\lambda>$ $14.2 \mu \mathrm{m}$ prevents the determination of [F1000W]-[F1500W] and $[\mathrm{F} 1000 \mathrm{~W}]-[\mathrm{F} 2100 \mathrm{~W}]$ for the majority of these objects (those indicated with open pentagons in the top panels).

\subsection{Low-mass dusty M-type AGB stars}

The magenta triangles in Fig. 4 indicate stars that we interpret as low-mass dusty stars. The evolutionary tracks of these objects in the different observational planes overlap substantially, mainly because of the similar luminosities, favoured by the occurrence of core electron degeneracy for $M \leqslant 2 M_{\odot}$ progenitors.

The redwards extension of the tracks depends on both the initial mass and the metallicity. Stars of initial mass $M \lesssim 1.5 M_{\odot}$ reach higher degrees of obscuration during the phases preceding the C-star stage, compared to their $2-3 M_{\odot}$ counterparts (see discussion in Sect. 2.2). The lower the metallicity the shorter the redwards extension of the evolutionary tracks, owing to the lower amounts of silicon present in the envelope of low $\mathrm{Z}$ stars. This behaviour with mass and chemical composition is discussed in detail in Dell'Agli et al. (2015a, Fig. 8). The position of this class of objects in the various planes in mainly determined by the optical depth, which allows us to draw theoretical obscuration sequences, indicated with solid lines in Fig. 4.

The SED of the stars indicated with magenta triangles confirms the theoretical expectations, as it is reproduced by assuming a majority $(70-90 \%)$ of silicate grains, with lower percentages of alumina dust and solid iron and luminosities in the range $5 \times 10^{3}-10^{4} L_{\odot}$ (see Table 2 for details). We report in Fig. 6 two examples of these objects, for which we show the Spitzer IRS data, the photometry available in the literature and the best fit model. These two cases exhibit significantly different degrees of obscuration that cover almost the whole range of the optical depths derived.

This range covered by the luminosities and the position of these stars in the PL plane shown in Fig. 5 suggests that these sources form a homogeneous group, made up by objects that descend from low-mass progenitors (in agreement with Trabucchi et al. 2018), the progeny of $M \leqslant 1.5 M_{\odot}$ stars, older than $\sim 2$ Gyr. Indeed stars of mass $2 M_{\odot} \leqslant$ 

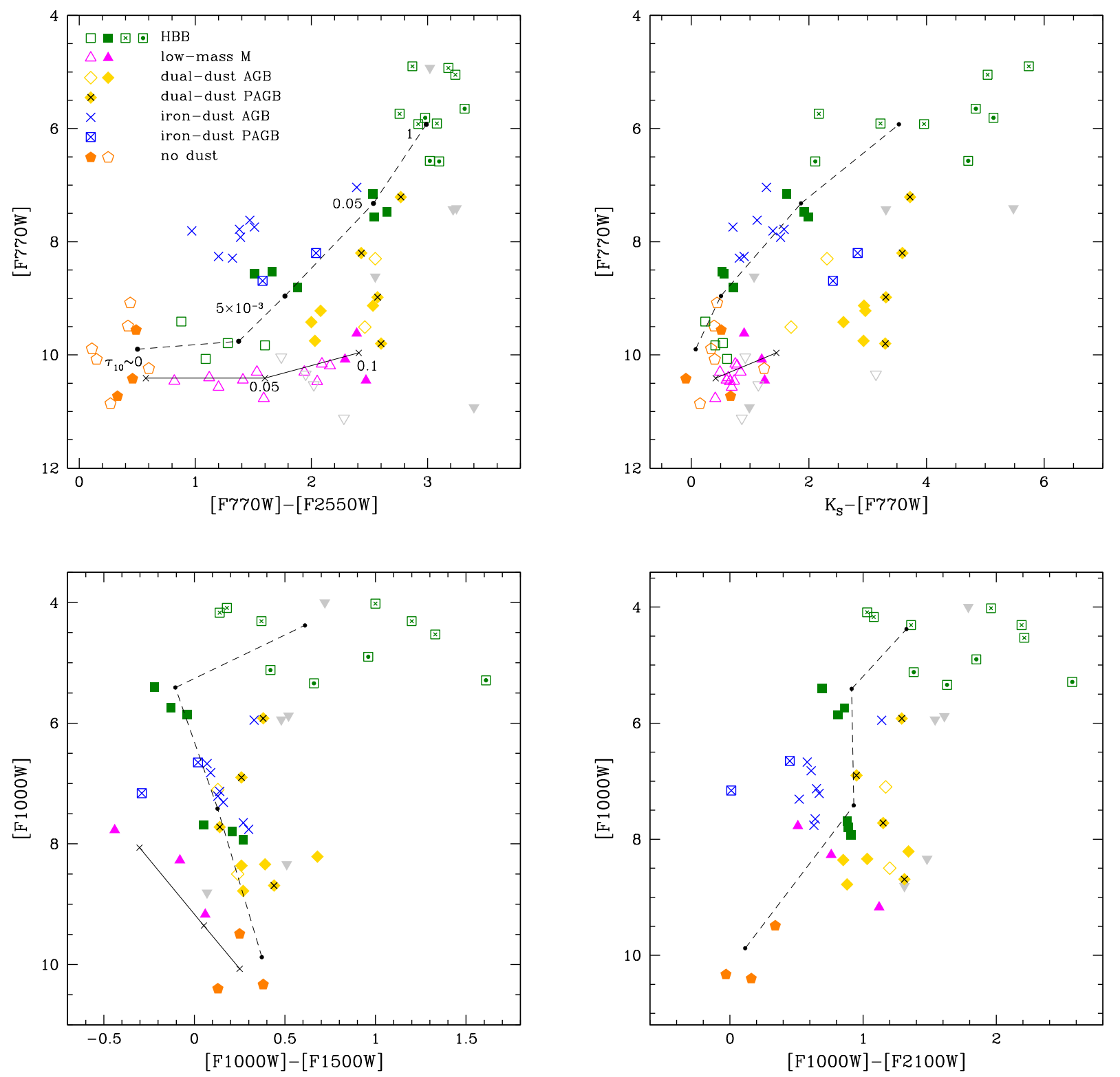

Figure 4. The distribution of the LMC stars belonging to the sample studied by Jones et al. (2012, 2014, 2017) in various observational planes, built with different combinations of the filters of the MIRI camera, mounted onboard of the JWST and 2MASS $K_{S}$ filter. The values of the magnitudes have been obtained by convolving Spitzer IRS data with the transmission curves of the various filters. For the stars indicated with open points, the IRS data were truncated to $\lambda \sim 14.2 \mu \mathrm{m}$; in these cases we used the MIPS [24] magnitude, when available, as a rough estimate of [F2550W], as the difference between them is only a few hundredth of magnitudes. The different symbols refer to the various classes of objects, according to the legend reported in the top, left panel. Among these, grey triangles indicate sources for which we could not provide a reliable interpretation on the basis of their SED. The solid and dashed lines indicate the obscuration sequence of low-mass, oxygen-rich AGB stars and of massive AGBs experiencing HBB, respectively (see Sect. 4.2 and 4.3 for details). The black points along the two sequences refer to typical values of the optical depth $\tau_{10}$, which are indicated in the top, left panel.

$M \leqslant 3 M_{\odot}$ form negligible quantities of dust during the M-stage (see discussion in Sect. 2.3).

In summary, we base the characterization of the stars in this sub-sample on the following points: i) the luminosities are below $\sim 10^{4} L_{\odot}$; ii) dust is mainly composed of silicates; iii) the optical depths are in the range $0.005<\tau_{10}<0.1$.

The range of optical depths and dust composition re- quired to reproduce the SED are in agreement with the lowmass models discussed in Dell'Agli et al. (2015a). The fraction of silicates is found to increase across the obscuration sequence, ranging from $\sim 70 \%$ to $\sim 90 \%$. Alumina dust is more stable than silicates, thus it forms at larger rates, in a more internal region of the circumstellar envelope, at temperatures of the order of $\sim 1400 \mathrm{~K}$; silicates form in a more 


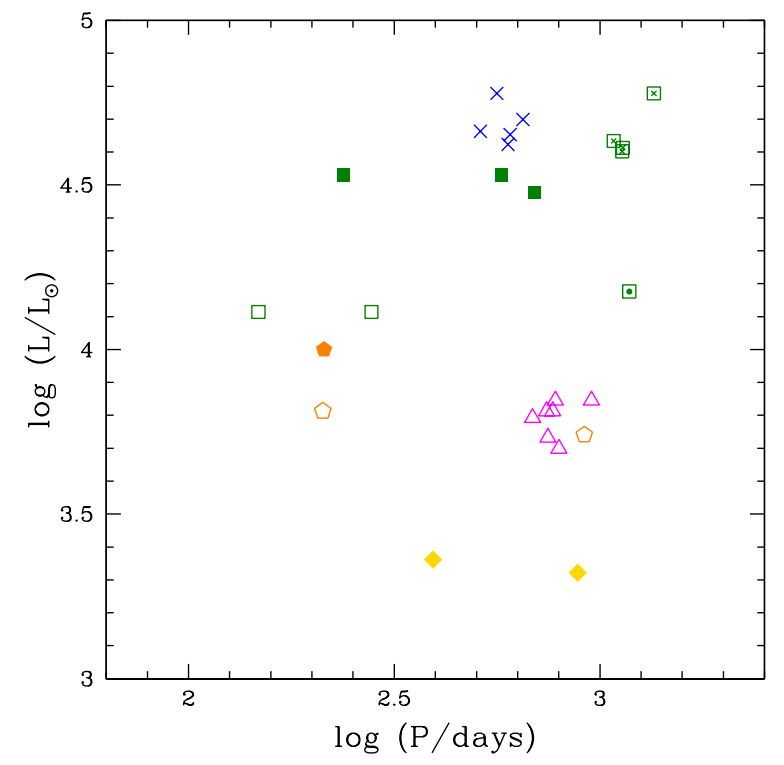

Figure 5. Period - Luminosity diagram of the LMC M-type stars in the sample for which OGLE periods are available. Luminosities are those reported in Table 2. Symbols are the same used in Fig. 4.

external zone, at temperatures $T \sim 1100 \mathrm{~K}$ (Dell'Agli et al. 2014a). The increase in wind density triggers a higher formation rate of both species; however, this has a larger effect on the amount of silicates formed, as alumina dust is so stable that it forms in quantities close to saturation, thus being less sensitive to variations in the thermodynamics of the wind.

The reddest objects of this group among the obscuration sequence in the ([F770W]-[F2550W], [F770W]) plane, with $[\mathrm{F} 770 \mathrm{~W}]-[\mathrm{F} 2550 \mathrm{~W}]>2$ and $\tau_{10} \sim 0.1$, are evolving through the final phases as $\mathrm{M}$ stars, before becoming carbon stars. This hypothesis was proposed by Dell'Agli et al. (2015a) to explain the O-rich stars studied by Blum et al. (2006), that define a prominent finger in the colourmagnitude ([8.0]-[24],[24]) diagram.

The present interpretation might be tested by measuring the surface $\mathrm{C} / \mathrm{O}$ ratio in these stars (e.g. via near-IR spectroscopy), that is expected to increase across the sequence, until becoming slightly below unity for the sources exhibiting the largest degree of obscuration.

In this context, the sources SSID 22 and SSID 130, indicated with yellow open diamonds, represent an ideal prosecution of this evolutionary path, because we interpret them as stars that have only recently reached the C-star stage, with the circumstellar envelope hosting a more internal, hot, dusty layer, populated by solid carbon particles, and a cooler zone, where silicate dust formed during previous evolutionary phases is expanding away from the star. The details of the best fit obtained for SSID 22 are shown in Fig. 7 .

The obscuration trend in the ([F770W]-[F2550W], $[\mathrm{F} 770 \mathrm{~W}])$ plane is approximately horizontal; this behaviour is connected with the evolution of the shape of the SED of low-mass stars with $\tau_{10}$, shown in the left panel of Fig. 2. With increasing optical depth the height of the silicate fea- ture and the overall spectrum in the $\lambda>10 \mu \mathrm{m}$ region increase, whereas the $[\mathrm{F} 770 \mathrm{~W}]$ flux keeps approximately constant, owing to the reasons explained above, related to the shape of the silicate feature.

Inspection of Fig. 4 shows that ([F770W]-[F2550W], $[\mathrm{F} 770 \mathrm{~W}])$ is the only plane where the obscuration sequence of these objects has a significant extension and does not overlap with different kind of stars. In the $\left(K_{S^{-}}[\mathrm{F} 770 \mathrm{~W}]\right.$, $[\mathrm{F} 770 \mathrm{~W}])$ plane they lay very close to the dust-free sources, for the reasons given in Sect. 4.1. In the ([F1000W]$[\mathrm{F} 1500 \mathrm{~W}],[\mathrm{F} 1000 \mathrm{~W}])$ plane these sources populate a diagonal band, reported in the bottom, left panel of Fig. 4, with the most obscured stars located in the bluer and brighter region. This is due to the increasing prominence of the $10 \mu \mathrm{m}$ feature, that rises the $[\mathrm{F} 1000 \mathrm{~W}]$ flux. The colour range, $\Delta([\mathrm{F} 1000 \mathrm{~W}]-[\mathrm{F} 1500 \mathrm{~W}]) \sim 0.5 \mathrm{mag}$, is less extended than in the previous cases, because the increase in the flux in the $10 \mu \mathrm{m}$ spectral regions is accompanied by the rise of the flux in the whole region at wavelengths $\lambda>10 \mu \mathrm{m}$ (see left panel of Fig. 2).

In the ([F1000W]-[F2100W], [F1000W]) diagram the identification of these stars is even harder, because the percentage increase in the flux as the optical depth increases are very similar in the spectral regions where the two filters are centered, i.e. 10 and $21 \mu \mathrm{m}$.

\subsection{Stars undergoing hot bottom burning}

We now turn the attention to the stars indicated with green squares in Fig. 4. A few examples of the SED of these objects are shown in Fig. 8, where we present the observations and our corresponding theoretical SED.

Unlike low-mass stars, massive AGBs span a wide range of luminosities, extending by a factor $\sim 5$ (see left panel of Fig. 1). The position of each model in the various planes is determined by the values of the luminosity and optical depth. Therefore the tracks of stars of different masses are practically overlapped, the only difference being that the higher the mass of the progenitor the larger the maximum luminosity and degree of obscuration reached (see left panel of Fig. 1), and the wider the excursion of the evolutionary track in the planes. The dashed lines shown in Fig. 4 connect points characterized by different optical depths and luminosities, therefore can be considered as obscuration sequences of massive AGBs.

The extension of the obscuration sequences is at first order independent of metallicity. On the other hand, on the statistical side, we must consider that for lower metallicities the range of masses that reach the largest degrees of obscuration is narrower and, more important, the largest optical depths are experienced only during a small fraction of the AGB life (see Fig. 2 in Dell'Agli et al. 2015b, for the metallicity effects on the obscuration of massive AGBs).

The luminosities deduced from the analysis of the SED are in the range $2-8 \times 10^{4} L_{\odot}$ and the optical depths span the interval $\tau_{10} \sim 0.005-3$. The SED of these sources can be nicely interpreted by assuming a "standard" dust mineralogy for obscured M stars: a dominant contribution from silicates, with percentages in the range $60-100 \%$, completed by smaller fractions of alumina dust and solid iron. The higher the required fraction of silicate the larger the 

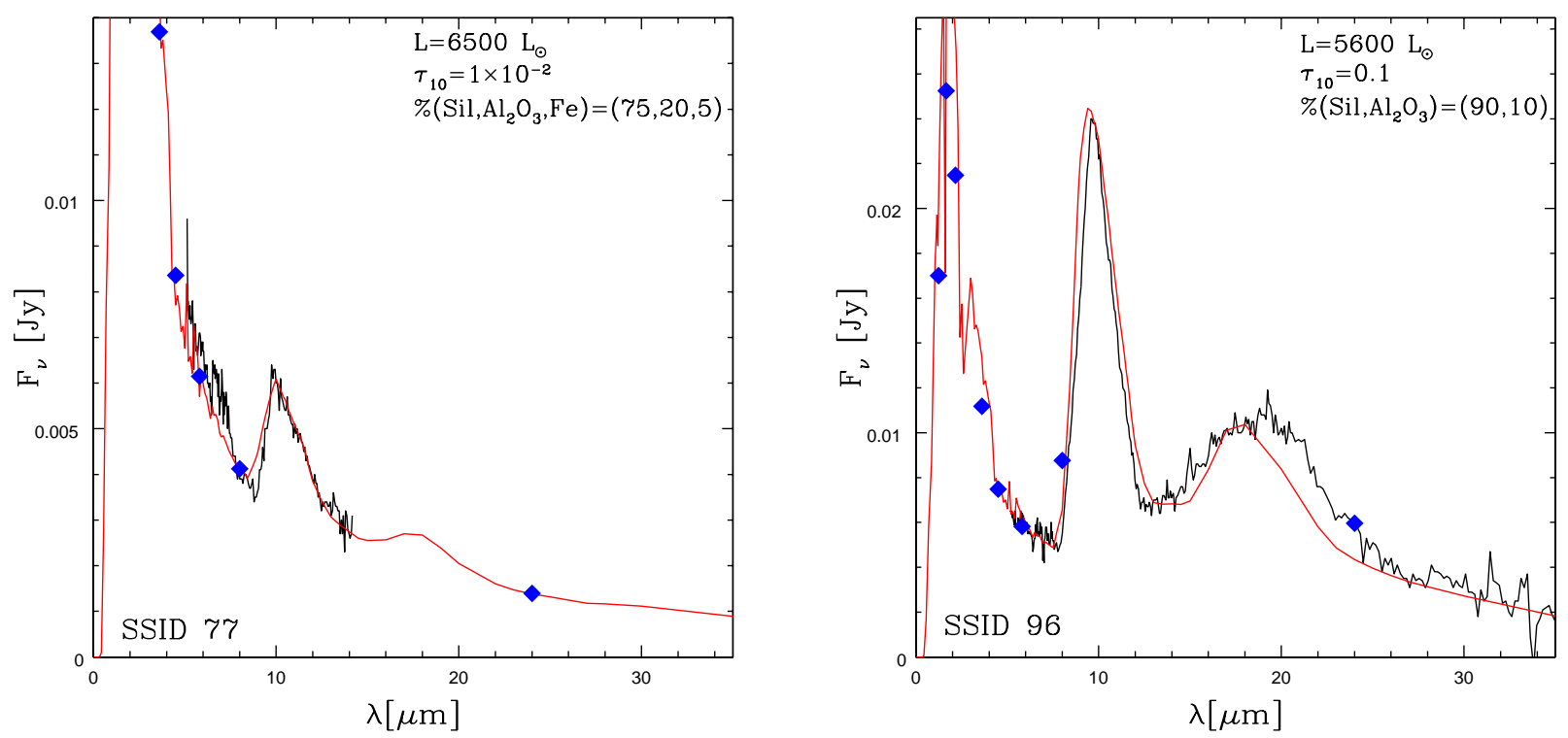

Figure 6. The Spitzer IRS spectra (black line) of two stars, selected among those interpreted as low-mass AGBs, taken along the obscuration sequence of low-mass stars shown in Fig. 4. The photometry is indicated with blue diamonds. The best fit, indicated with the red line, is obtained by assuming the luminosity, dust composition and optical depth indicated in the two panels.

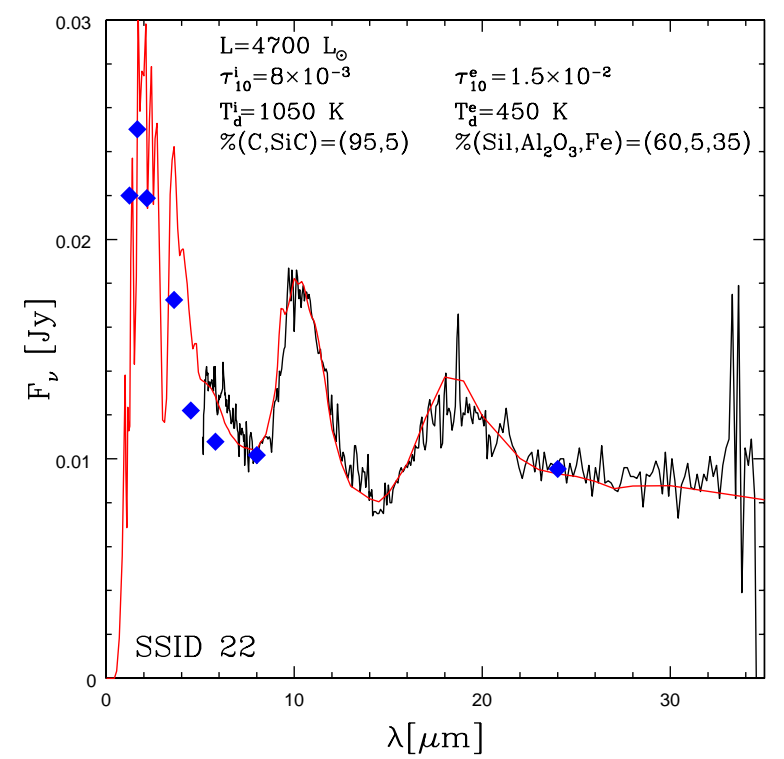

Figure 7. Best-fit model for the source SSID22 (same symbols and colours as in Fig. 6 are used), interpreted as a low-mass star that has just reached the C-star stage (see text for details). The values of the overall luminosity and of the parameters of the internal and external dust layers are indicated.

optical depth, in agreement with the arguments presented earlier in this section.

These results, compared with the theoretical models discussed in Sect. 2 (see left panel of Fig. 1 and the se- quence of SEDs in the right panel of Fig. 2), indicate that these stars descend from progenitors of mass above $3 \mathrm{M} \odot$, formed not earlier than $\sim 250-300 \mathrm{Myr}$ ago. This is also confirmed by their position in Fig. 5: their PL relation is in agreement with Trabucchi et al. (2018) models for massive $\operatorname{AGB} \operatorname{stars}\left(>3 M_{\odot}\right)$.

The significant amount of dust present in the circumstellar envelope rules out the possibility that these stars are metal poor and suggests that their metallicity is $Z \sim$ $4-8 \times 10^{-3}$; this hypothesis is supported by the massmetallicity relation of the LMC, according to which star formation in the last $1 \mathrm{Gyr}$ is dominated by a $Z=8 \times 10^{-3}$ stellar population (Harris \& Zaritsky 2009).

\subsubsection{Massive AGBs in the colour-magnitude planes}

The afore mentioned obscuration sequence defined by massive AGB stars can be used to attempt their characterization. Generally speaking, we find that the optical depths increase across the sequences in the different planes: the sources indicated with open and full green squares are characterized by $5 \times 10^{-3}<\tau_{10}<0.1$, whereas dotted and crossed green squares correspond to $\tau_{10}>0.5$.

In the ([F770W]-[F2550W], [F770W]) plane (see top, left panel of Fig. 4) the region populated by these objects extends over $\sim 2 \mathrm{mag}$ in [F770W]-[F2550W] and almost 4 mag in $[\mathrm{F} 770 \mathrm{~W}]$. Unlike the lower mass counterparts, discussed in Sect. 4.2, the luminosity is generally correlated with $\tau_{10}$, the brighter stars being on the average more obscured. The stars indicated with green, dotted squares are an exception to this rule, as they are fainter than their counterparts with similar degree of obscuration, indicated with green, crossed squares. We will discuss these sources later in this section. 

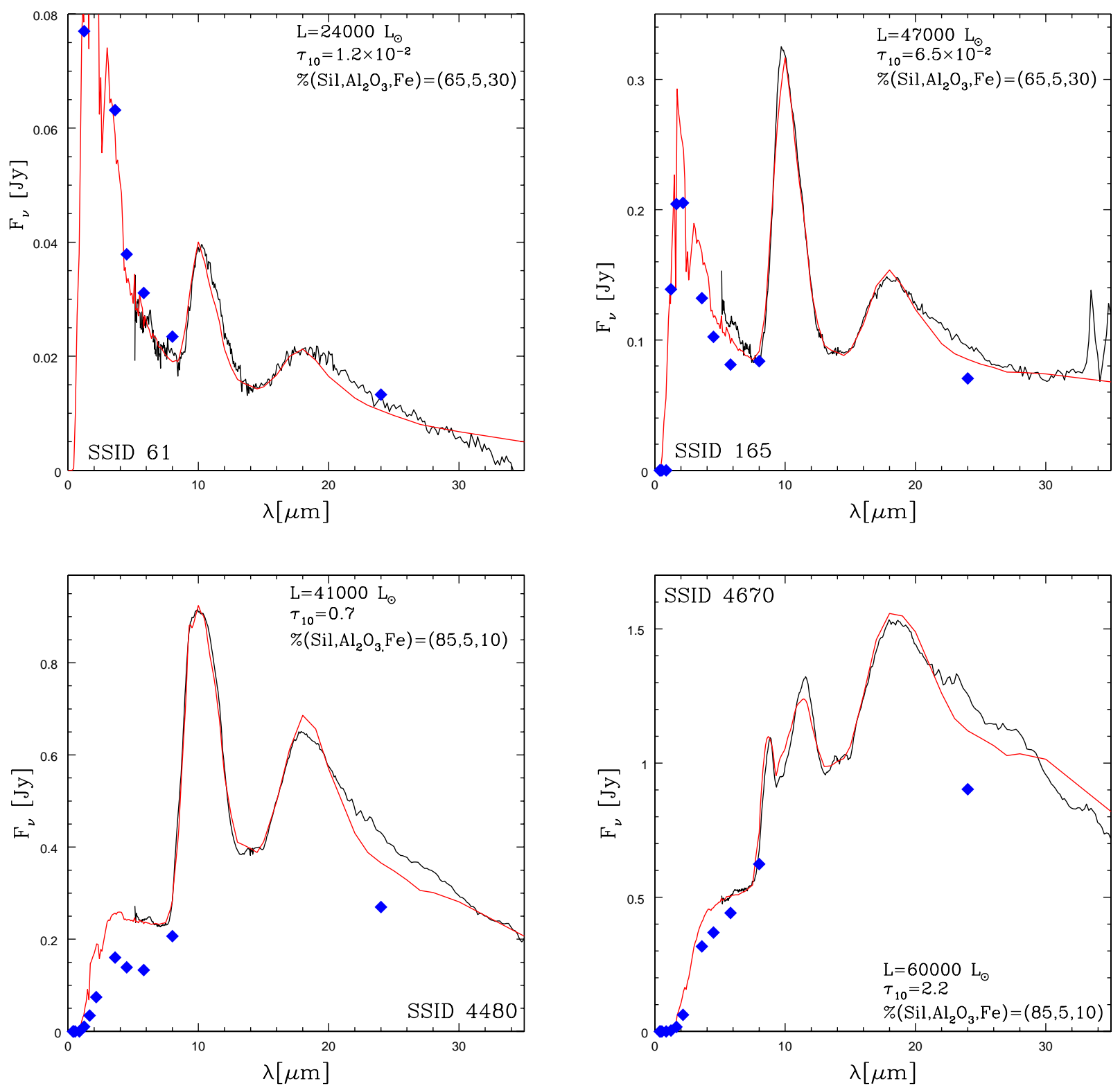

Figure 8. The Spitzer IRS spectrum (black line) of 4 stars, selected among those interpreted as massive AGBs, currently experiencing HBB, taken along the obscuration sequence indicated with a dashed line in Fig. 4 . The photometry and the best-fit are shown, with the parameters adopted.

Both the $[\mathrm{F} 770 \mathrm{~W}]$ and $[\mathrm{F} 2550 \mathrm{~W}]$ fluxes rise with increasing $\tau_{10}$, as shown in the right panel of Fig. 2 and in the examples in Fig. 8. On the other hand, it is clear from Fig. 2 that the increase of the flux in the spectral region around $25.5 \mu \mathrm{m}$ is percentually larger when compared to the $\lambda \sim 7.7 \mu \mathrm{m}$ zone, thus making [F770W]-[F2550W] to increase with $\tau_{10}$, provoking a rightwards trend of the obscuration sequence.

The stars discussed here trace a diagonal pattern in the $\left(K_{S^{-}}\right.$[F770W], [F770W]) plane. With the excursion in the [F770W] magnitude discussed before, $K_{S^{-}}[\mathrm{F} 770 \mathrm{~W}]$ spans a range of almost 6 mag, due to the gradual shift of the whole
SED towards mid-IR wavelengths and the decrease in the near-IR flux, particularly relevant for $\tau_{10}>0.1$. This allows for a higher sensitivity to the degree of obscuration, although the measurement of the near-IR flux could be critical for the most obscured stars. Indeed one out of the 3 brightest stars are not reported in this plane, owing to the lack of the $K_{S}$ flux.

Regarding the ([F1000W]-[F1500W], [F1000W]) diagram, shown in the bottom, left panel of Fig. 4, the path traced by the obscuration pattern presents a turning point: the initial trend towards the blue, down to [F1000W]$[\mathrm{F} 1500 \mathrm{~W}] \sim-0.2$, is followed by a redwards excursion. The 
first part is due to the appearance of the feature at $9.7 \mu \mathrm{m}$, that rises the $10 \mu \mathrm{m}$ flux and diminishes the emission in the wavelength region around $15 \mu \mathrm{m}$ (see Fig. 2). When $\tau_{10}$ exceeds $\sim 0.1$ this trend is reversed, because the whole mid-IR flux is lifted. The most obscured stars distribute approximately horizontally on this plane, because for $\tau_{10}>2$ the silicate feature turns into absorption, thus no further increase in the $10 \mu \mathrm{m}$ flux occurs. The stars with the highest degree of obscuration that correspond to the brightest and youngest objects discussed previously, are located in the right, upper region of the plane.

In the ([F1000W]-[F2100W],[F1000W]) diagram the sequence of the stars discussed here follows a slightly different behaviour compared to the ([F1000W]-[F1500W],[F1000W]) plane. The obscuration pattern extends towards higher [F1000W] fluxes; however, no clear turning point is found, because the rise of the SED in the 10 and $21 \mu \mathrm{m}$ spectral regions occur with similar percentages. The stars with $\tau_{10}>0.5$ populate the brightest region of this plane, at $[\mathrm{F} 1000 \mathrm{~W}]-[\mathrm{F} 2100 \mathrm{~W}]>1$.

\subsubsection{Stars undergoing soft $H B B$}

The stars indicated with open squares harbour little amounts of dust in their circumstellar envelope. The optical depths are a few $10^{-3}$. According to our interpretation, they have just started the HBB activity. We expect that only a modest depletion of the overall surface carbon has occurred and that the ${ }^{12} \mathrm{C} /{ }^{13} \mathrm{C}$ ratio has dropped to values close to the equilibrium abundances, of the order of $\sim 4$. No meaningful depletion of the surface oxygen is expected.

Following the theoretical obscuration pattern, we analyze the sources indicated with full squares in Fig. 4, that have $0.01<\tau_{10}<0.1$ and luminosities covering the interval $2-4.5 \times 10^{4} L_{\odot}$. The sources SSID 61 and SSID 165, reported in the top panels of Fig. 8, belong to this group. The luminosities and $\tau_{10}$ 's given above suggest that these objects are the progeny of $4-5 M_{\odot}$ stars formed $100-300$ Myr ago, currently experiencing HBB. As shown in the middle panel of Fig. 1, the surface carbon should be $\sim 10-20$ timessmaller than in their less obscured counterparts, the surface nitrogen enhanced by one order of magnitude and the surface ${ }^{12} \mathrm{C} /{ }^{13} \mathrm{C}$ ratio should be very close to the equilibrium value, i.e. $3-4$. Furthermore, these stars are expected to be experiencing the Cameron-Fowler (1971) mechanism, thus they should be enriched in lithium.

\subsubsection{Highly obscured O-rich AGBs}

The objects indicated with crossed and dotted green squares in Fig. 4 are producing dust at a high rate, as confirmed by the large optical depths, $\tau_{10}>0.5$.

In the $([\mathrm{F} 770 \mathrm{~W}]-[\mathrm{F} 2550 \mathrm{~W}],[\mathrm{F} 770 \mathrm{~W}])$ and $\left(K_{S^{-}}\right.$ $[\mathrm{F} 770 \mathrm{~W}],[\mathrm{F} 770 \mathrm{~W}])$ planes the obscuration patterns are most easily distinguished. The stars with $[\mathrm{F} 770 \mathrm{~W}]>5.5$ (all the crossed and dotted, green squares, but the 3 brightest objects) have luminosities below $\sim 5 \times 10^{4} L_{\odot}$; an example is the source SSID 4480, reported in the bottom, left panel of Fig. 8. Based on the results shown in the left panel of Fig. 1, and on the discussion in Sect. 2.1, we deduce that they descend from progenitors of mass in the range $3.5-5 M_{\odot}$, formed between $100 \mathrm{Myr}$ and $300 \mathrm{Myr}$ ago. The optical depths, $0.5<\tau_{10}<1.5$, are consistent with this conclusion.

The 3 brightest sources with $[\mathrm{F} 770 \mathrm{~W}]<5.5$ are characterized by a high degree of obscuration, with $\tau_{10} \sim 2-3$, and luminosities above $5 \times 10^{4} L_{\odot}$, that, based on the results shown in the left panel of Fig. 1, indicate progenitors of mass above $\sim 5 M_{\odot}$, younger than $\sim 100 \mathrm{Myr}$.

A word of caution regarding dust formation modelling in the winds of these peculiar objects is needed here. While AGB models reproduce both the luminosities and the periods of these sources, the optical depths required, in the range $2<\tau_{10}<3$ are higher than the largest theoretical values predicted, of the order of $\tau_{10} \sim 1$. This outlines some tension between models and observations. Recent radiationhydrodynamic (RHD) models of winds of M-type AGB stars (see, e.g., Höfner et al. 2016; Höfner \& Olofsson 2018) indicate that dust formation will indeed start deeper inside the atmosphere (at smaller condensation radius), but this result was obtained at solar metallicity (not typical LMC metallicity). However, the difference in condensation radii in the simple stationary outflows of the stellar evolution models and the more sophisticated RHD models, is likely a result of the different levels of detail of the two types of models. First, the RHD models are known to produce different results due to the dynamics (pulsation) and time dependence. Second, frequency-dependent radiative transfer (RT) changes the energy balance and temperature structure compared to the atmospheric structures obtained in the stellar evolution models. That is, the simplistic atmosphere models we employ probably do not predict correct condensation radii.

\subsubsection{A class of massive $A G B s$ in the very late $A G B$ phases?}

A second issue of the present understanding is the interpretation of the stars indicated with dotted, green squares in Fig. 4. These sources have luminosities of the order of $2 \times 10^{4} L_{\odot}$, significantly smaller than those of their brighter counterparts, indicated with crossed, green squares. These luminosities are compatible both with those of stars evolving through the initial TPs, or at the end of their AGB life, after HBB was turned-off. Their periods (we refer to the OGLE period for SSID 4007 and those from GS18 for the other sources in this group) are close or above $1000 \mathrm{~d}$. Because in the first case we should expect periods below $\sim 500 \mathrm{~d}$ (see Fig. 5), we believe more plausible that these stars are experiencing the latest AGB phases.

The main drawback of this interpretation is that their degree of obscuration is also expected to be low (see the bottom, left panel of Fig. 4), whereas their optical depths are $\tau_{10} \geqslant 1$. A possible solution is that during the very final AGB phases the formation of a disc favours the accumulation of the dust produced during earlier phases, such that the overall dust density is significantly higher than predicted by the simplified description of the wind used in the present analysis. We leave this problem open.

The surface chemical composition of the stars indicated with crossed and dotted, green squares is expected to show the imprinting of proton-capture processing, in analogy with the less obscured counterparts, indicated with open and full, green squares. These stars are also expected to have started 
the Ne-Na nucleosynthesis, with a sodium enrichment by a factor of $\sim 5$ (Ventura et al. 2013). If this small sample includes stars of lower metallicity, of the order of $Z=4 \times 10^{-3}$, then we would observe the results of oxygen burning and of $\mathrm{Mg}-\mathrm{Al}$ nucleosynthesis, with a surface $\mathrm{Al}$ enhancement by a factor $5-10$ and oxygen depletion by a factor $\sim 2$ (Ventura et al. 2016).

\subsection{Iron-dust stars}

\subsubsection{Massive AGB stars with iron dust}

The sample studied by Jones et al. (2012, 2014, 2017) include a paucity of bright objects, indicated with blue crosses in Fig. 4 , whose luminosities are above $4.5 \times 10^{4} L_{\odot}$. The large fluxes indicate that these stars are the progeny of $M>4 M_{\odot}$ stars, currently experiencing HBB. The peculiarity of these stars is in their SED, that exhibits the main silicate feature at $9.7 \mu \mathrm{m}$, and cannot be reproduced by assuming a silicatedominated dust mineralogy. An example of such a SED is shown in the left panel of Fig. 9. Unlike the bright stars undergoing $\mathrm{HBB}$, discussed earlier in this section, the degree of obscuration is not particularly large, with $\tau_{10}<0.01$.

Marini et al. (2019a) proposed that the stars in this group are the progeny of metal-poor, $M>4 M_{\odot}$ stars, formed $\sim 100 \mathrm{Myr}$ ago. The little amount of dust present in their surroundings is due to the low metallicity, of the order of $Z \sim 10^{-3}$. According to Marini et al. (2019a) the peculiar SED is related to the strong HBB experienced, that provoked a significant drop in the surface abundances of oxygen and magnesium, and the consequent shortage of water molecules and of magnesium atoms, both essential ingredients to form silicate particles. The mineralogy of the dust around these stars is therefore dominated by solid iron, the latter species being not affected by HBB. Iron grains account for $\sim 80 \%$ of the total dust, with smaller percentages of silicates $(\sim 15 \%)$ and alumina dust $\sim 5 \%$.

As shown in Fig. 4, in the ([F770W]-[F2550W], $[\mathrm{F} 770 \mathrm{~W}])$ plane these objects populate the region at $1<$ $\left[\right.$ F770W]-[F2550W] $<1.5$ and $[\mathrm{F} 770 \mathrm{~W}] \sim 8^{7}$. The colours of these sources are not extremely red, consistently with the low degree of obscuration. The $[\mathrm{F} 770 \mathrm{~W}]$ fluxes are higher than those exhibited by the more metal rich counterparts of similar colours. As discussed in Marini et al. (2019a), this is due to the peculiar shape of their SED, because the low percentage of silicates prevents the depression of the flux in the spectral region around $8 \mu \mathrm{m}$, thus lifting the $[\mathrm{F} 770 \mathrm{~W}]$ emission.

In the $\left(K_{S}-[\mathrm{F} 770 \mathrm{~W}], \quad[\mathrm{F} 770 \mathrm{~W}]\right)$ and $([\mathrm{F} 1000 \mathrm{~W}]-$ [F1500W], [F1000W]) planes (see Fig. 4) the iron dust stars cannot be easily identified, as they lie along the obscuration sequence of their counterparts of higher metallicity, close to those indicated with full, green squares.

In the ([F1000W]-[F2100W], [F1000W]) plane these sources populate a zone $\sim 0.2 \mathrm{mag}$ bluer than the stars with similar optical depths and higher metallicity: this is because

\footnotetext{
7 The only exception is the source SSID 4098, with $[F 770 W]-$ $[F 2550 \mathrm{~W}] \sim 2.4$, which is evolving through a less advanced evolutionary phase, when oxygen in the envelope has not been completely burnt, so that $\sim 40 \%$ of dust is made up of silicates.
}

the higher fraction of iron dust affects the SED by decreasing the relative height of the secondary silicate feature, at $\sim 20 \mu \mathrm{m}$, which diminishes the $[\mathrm{F} 2100 \mathrm{~W}]$ flux, rendering the [F1000W]-[F2100W] colours bluer.

\subsubsection{Post-AGB stars with iron dust}

In the family of stars surrounded by iron dust we also consider SSID 4267 and SSID 6, indicated in Fig. 4 with open, blue squares filled with crosses. The Spitzer IRS and photometric data and the best-fit model for SSID 4267 are shown in the right panel of Fig. 9.

We find that these two objects have effective temperatures in the range $T_{\text {eff }} \sim 7000-8000 \mathrm{~K}$, luminosities $\sim 2-2.5 \times 10^{4} L_{\odot}$, and are surrounded by dust, mainly composed by solid iron. These parameters point to an evolutionary nature that is more evolved than that of stars in the AGB phase, suggesting that they are post-AGBs; this is also consistent with the large IR excess observed (van Winckel 2003; van Aarle et al. 2011; Kamath et al. $2014,2015)$ and with the predicted post-AGB luminosities of metal-poor massive AGBs (Miller Bertolami 2016). SSID 4267 is classified as a post-AGB candidate in the photometric study to search for post-AGB stars in the LMC by van Aarle et al. (2011). Additionally, in the Spitzer and TIMMI2 study by Gielen et al. (2011a) of post-AGB disc sources, SSID 6 is classified as a post-AGB star with atypical dust chemistry, because it shows no strong evidence for the expected crystalline features in their spectra. A combination of amorphous silicate dust and alumina was required to fit the spectral features in the $5 \mu \mathrm{m}<\lambda<40 \mu \mathrm{m}$ region. Since we find that SSID 4267 and SSID 6 are surrounded by irondust, we classify these two objects as iron-dust post-AGB stars.

Iron dust post-AGB stars are characterized by optical depths $\tau_{10} \sim 1-5 \times 10^{-3}$, smaller than their counterparts evolving through the AGB phase. This is because dust formation stops when the stars leave the AGB and the dust layer currently observed is expanding away from the central star, in a lower density region. The dust temperatures required, of the order of $700-900 \mathrm{~K}$, support this interpretation.

In the study by Woods et al. (2011) these objects have been classified as M-type AGB stars. Woods et al. (2011) identify post-AGB stars based on the presence of a distinguished double peak in the SED, separated by a clear minimum at $\lambda \sim 2 \mu \mathrm{m}$. SSID 4267 and SSID 6 do not show the expected minimum. Our interpretation for the lack of the expected minimum is that the presence of iron dust makes the minimum in the SED to vanish almost completely, thus rendering the spectrum similar to a scarcely obscured M-type AGB star. Such a modification of the SED is the typical effect of the presence of significant quantities of a featureless dust species (like solid iron is), as discussed in the analysis of dual-dust chemistry AGB and post-AGB stars studied by Marini et al. (2019b).

Furthermore, a meaningful difference between the SED of AGB and post-AGB stars surrounded by iron dust is the peak in the spectral emission, located at shorter wavelenghts in the latter case (see Fig. 9, right panel). As a consequence, AGB stars have lower [F770W] magnitudes than their postAGB counterparts, and their ([F770W]-[F2550W]) colours 

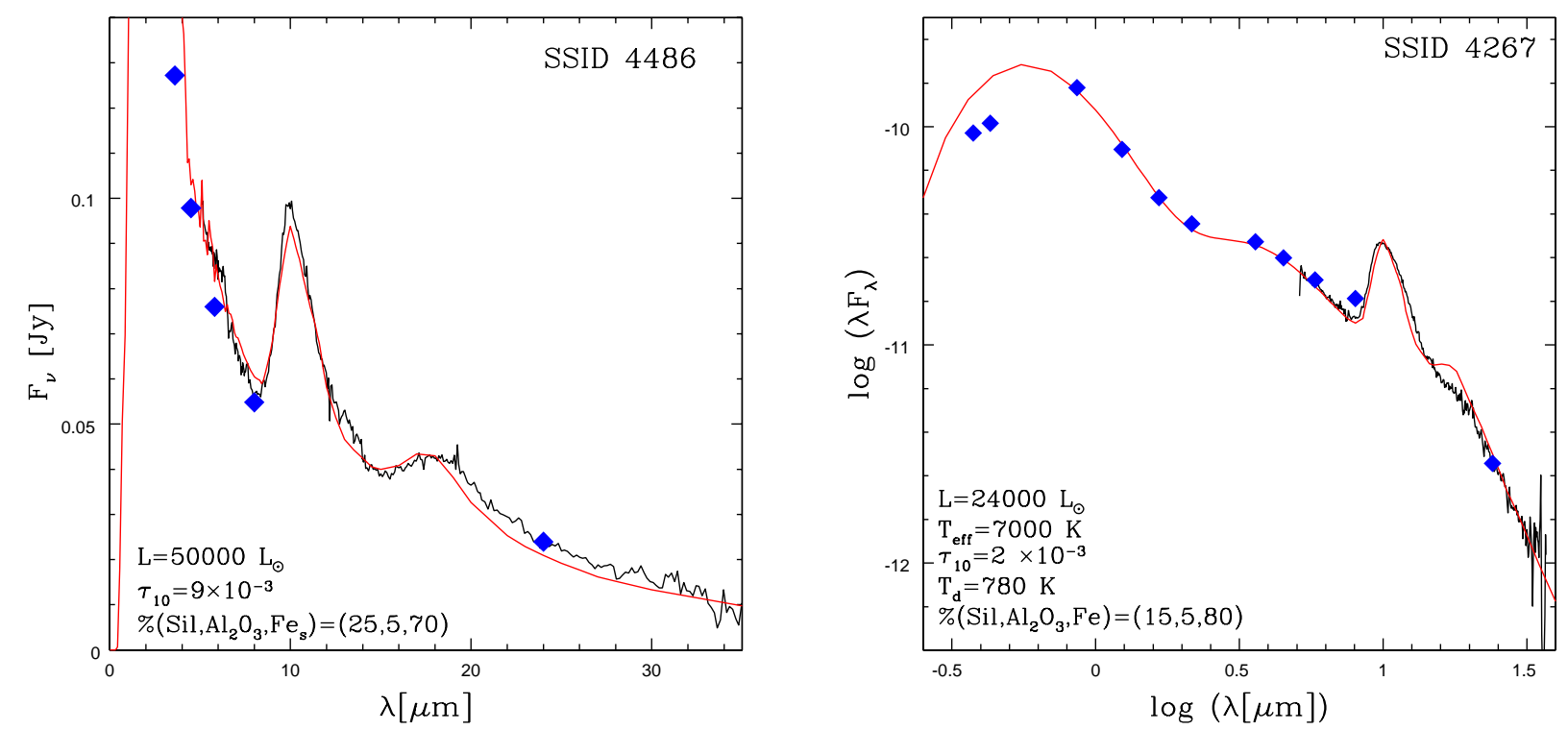

Figure 9. IRS, photometry results and best-fit models for the sources SSID 4486 (left panel) and SSID 4267 (right), interpreted as stars surrounded by dust, whose composition is dominated by iron grains, evolving through the AGB and the post-AGB phase, respectively. For SSID 4267 we report the flux in the $\log \left(\lambda F_{\lambda}\right)$ scale, to better understand why this object was misclassified as an AGB star (see text for a discussion on this point).

are bluer. This can be seen in the relative position of the two groups of stars in the ([F770W]-[F2550W], [F770W]) plane.

$\left(K_{S^{-}}[\mathrm{F} 770 \mathrm{~W}],[\mathrm{F} 770 \mathrm{~W}]\right)$ is the plane where iron dust AGB and post-AGB stars can be separated more easily. This is because the SED of AGB stars generally peaks at wavelengths around $\sim 2 \mu \mathrm{m}$, that renders their $K_{S^{-}}[\mathrm{F} 770 \mathrm{~W}]$ colours significantly bluer than in the post-AGB case.

In the $([\mathrm{F} 1000 \mathrm{~W}]-[\mathrm{F} 1500 \mathrm{~W}], \quad[\mathrm{F} 1000 \mathrm{~W}])$ and $([\mathrm{F} 1000 \mathrm{~W}]-[\mathrm{F} 2100 \mathrm{~W}], \quad[\mathrm{F} 1000 \mathrm{~W}])$ planes iron dust stars populate the same regions.

\subsection{Dual dust chemistry AGB stars}

The stars indicated with yellow diamonds in Fig. 4 exhibit a peculiar SED. Two examples are shown in Fig. 10. While the presence of silicate dust is indicated by the $9.7 \mu \mathrm{m}$ and $18.7 \mu \mathrm{m}$ features, the SED can be hardly accounted for by assuming the presence of a single silicate rich dust layer. This lead Marini et al. (2019b) to propose a dual dust chemical composition for these sources. Despite according to our interpretation yellow crossed diamonds and full diamonds indicate two groups of stars that are evolving through different evolutionary phases, we discuss them in the same context, owing to the similarities in the mass and formation epoch of the progenitors and in the mineralogy of the dust in their circumstellar envelope.

\subsubsection{Dual-dust post-AGB stars}

The yellow, crossed diamonds (see Fig. 4) indicate stars (SSID 4054, SSID 4245, SSID 4547, SSID 4595) character- ized by a dual-dust chemistry, with an effective temperatures above $5000 \mathrm{~K}$ and luminosities in the range $5 \times 10^{3}-10^{4} L \odot$. These parameters make them compatible with the postAGB phase of low-mass (below $2 M_{\odot}$ ) progenitors (see Sect. 2.4). This interpretation is consistent with the works by van Aarle et al. (2011) and Kamath et al. (2015) who carried out a photometric and spectroscopic classification, respectively, of post-AGB stars in the LMC. These studies, based on the near-IR excess, classify these sources as likely binary post-AGB stars with a 'disc-type' SED.

We interpret the SED of these objects as composed by an internal dusty region, hosting solid carbon particles, and a cooler and more external zone, populated by silicates, alumina dust and solid iron. The evolutionary scenario proposed by Marini et al. (2019b) is that these stars became Cstars during their last TP, after evolving as M-stars during the whole AGB life. The two dusty layers, currently expanding away from the central star, are populated by particles formed before and after the last TP. This scenario can work only if these sources did not experience further TPs after becoming carbon stars. According to the discussion in Sect. 2.1 this is indeed a common behaviour of all the stars of mass $1.2 M_{\odot}<M<2 M_{\odot}{ }^{8}$. Therefore, we deduce that these objects formed between 1 and 4 Gyr ago.

Waters et al. (1998) first proposed that the circumbinary disc may be responsible for mixed chemistry: oxygenrich dust had been ejected by the AGB star in the past and stored in the binary disc, while more recent mass-loss was carbon-rich. This interpretation has since been supported

8 The lower limit is due to the fact that stars of initial mass below $\sim 1.2 M_{\odot}$ never become carbon stars. 

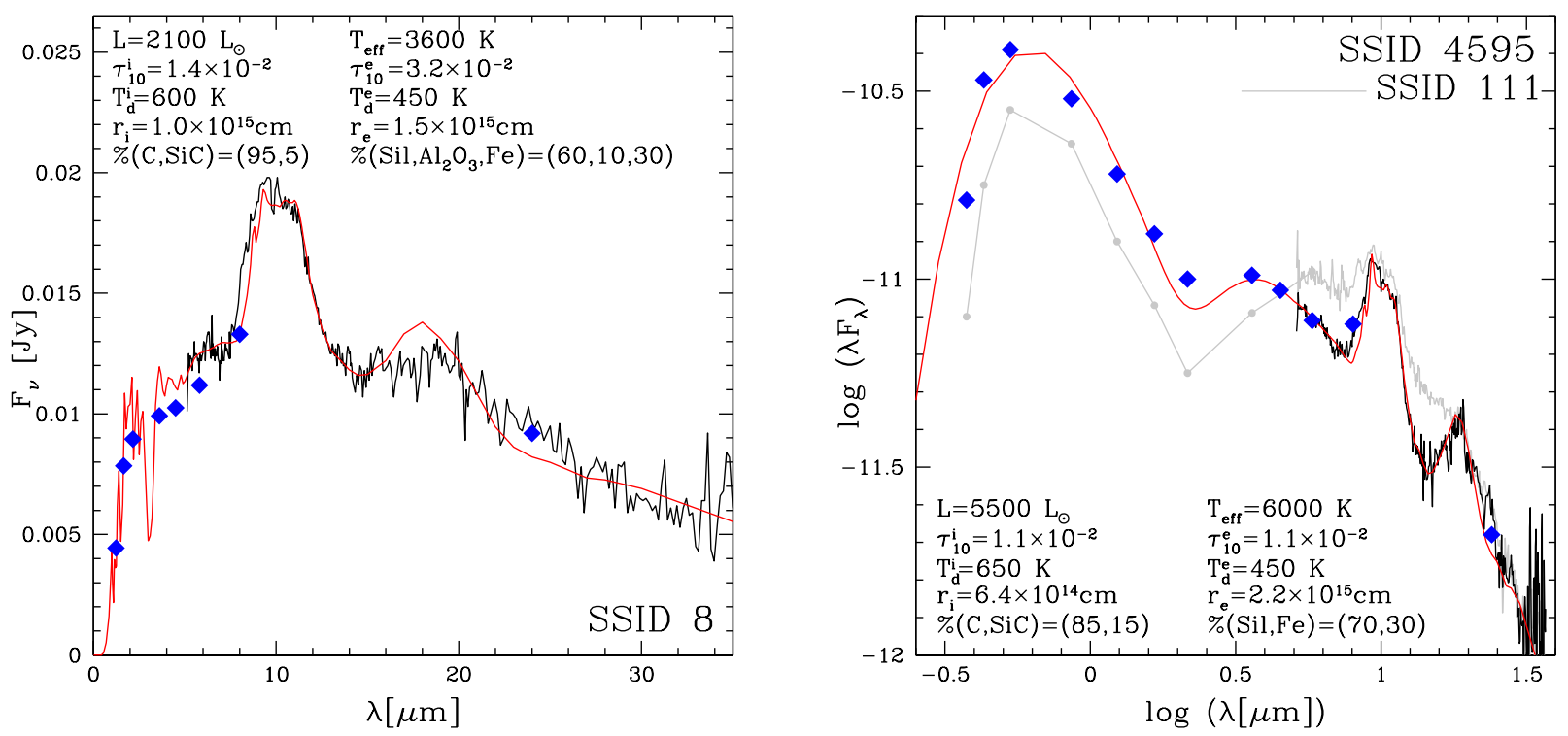

Figure 10. IRS, photometric data and best-fit model of two sources interpreted as stars surrounded by a double-chemistry dust, evolving through a post-thermal pulse phase (SSID 8, left) and the post-AGB phase (SSID 4595, right). The parameters of the two dust layers used to produce the best fit are indicated in the two panels. The right panel also shows (grey line) the spectrum of a source classified as post-AGB, following the criterion by Woods et al. (2011). It is clear the minimum at $\lambda \sim 2 \mu \mathrm{m}$, usually considered as an indicator of a post-AGB star.

by several studies including Gielen et al. (2011b); Matsuura et al. (2014).

Gielen et al. (2011a), based on the fit of the spectral region $5 \mu \mathrm{m}<\lambda<40 \mu \mathrm{m}$, concluded that the dust mineralogy for SSID 4595 and SSID 4547 is mainly composed of amorphous and crystalline silicates. However, we confirm that for the four dual-dust post-AGBs in this study, a significant fraction of carbon dust is required to allow the fit of the overall SED, especially in the $1 \mu \mathrm{m}<\lambda<8 \mu \mathrm{m}$ region. This is consistent with the detection of a weak PAH-like emission feature at $6.3 \mu \mathrm{m}$ in the SED of SSID 4547 (Marini et al. 2019b).

We note that PNe with a similar dual chemistry (i.e. PAHs + amorphous silicates + crystalline silicates) are rare but a few of them have been observed towards the Galactic Bulge (Perea-Calderón et al. 2009; Górny et al. 2010) ${ }^{9}$. As a confirmation of this, SSID 4547 was already observed and classified in Matsuura et al. (2014) as an oxygen-rich postAGB star with PAH features in its spectrum, raising the possibility that this object is characterized by a dual-dust chemistry.

\subsubsection{A class of faint AGBs with dual-dust}

Full, yellow diamonds in Fig. 4 point faint stars with luminosities in the range $2-4.5 \times 10^{3} L_{\odot}$. Their effective

9 Curiously, the PAHs in these few peculiar PNe are very similar to those in SSID 4547, where the PAHs at 6.3 (and $11.3 \mu \mathrm{m}$ ) are much stronger than the other two $(7.7$ and $8.6 \mu \mathrm{m}$, almost absent). temperatures indicate that they are AGB sources. It is not expected that such low-luminosity AGBs form dust in meaningful quantities (see Fig. 1).

The hypothesis that an unusually strong mass loss episode could have temporarily enhanced dust formation is unlikely, for the following reasons: i) the dust temperatures are cool, thus we are observing stars that are not producing dust in the present time; ii) the SED can be reproduced only if some carbon dust is added to silicates, alumina dust and solid iron.

In analogy with the post-AGB stars, Marini et al. (2019b) reproduced the observed SED claiming the presence of an internal dust zone, populated by carbonaceous particles and an outer layer, with a dust composition dominated by silicates. According to Marini et al. (2019b) these stars are evolving through a post-TP phase, during which the CNO nuclear activity is temporarily extinguished (which accounts for the low luminosities found), and no dust is being formed. The two dusty layers host dust grains formed during the phases immediately before the TP, when solid carbon grains were formed, and during the previous interpulse phase, before becoming carbon stars.

\subsubsection{Dual-dust stars in the colour-magnitude planes}

Among the different observational planes shown in Fig. 4, the diagram where dual-dust chemistry objects can be identified most easily is the $\left(K_{S^{-}}[\mathrm{F} 770 \mathrm{~W}],[\mathrm{F} 770 \mathrm{~W}]\right)$ plane. The presence of carbon dust makes the $[\mathrm{F} 770 \mathrm{~W}]$ flux higher and the $K_{S^{-}}[\mathrm{F} 770 \mathrm{~W}]$ colour bluer than in stars of similar optical depth. Therefore, the colours of these stars in this plane are comparable with those of the largely obscured stars dis- 
cussed in Sect. 4.3, that are much brighter. As shown in the top, right panel of Fig. 4, the stars with dual dust chemistry lie in the portion of the $\left(K_{S^{-}}[\mathrm{F} 770 \mathrm{~W}],[\mathrm{F} 770 \mathrm{~W}]\right)$ plane at $K_{S^{-}}[\mathrm{F} 770 \mathrm{~W}] \sim 2.5-3.5$, tracing an almost vertical sequence, below the obscuration pattern defined by the higher mass counterparts (indicated with green squares). In this $K_{S^{-}}[\mathrm{F} 770 \mathrm{~W}]$ colour range is located also SSID 130, that, on the other hand, is characterized by a dual dust chemistry as well (see Sect. 4.2).

In the ([F770W]-[F2550W], [F770W]) plane they lie slightly above the stars with similar degree of obscuration, again for the peculiar shape of the SED in the $\lambda \sim 8 \mu \mathrm{m}$ spectral region; however, their identification is more tricky than in the $\left(K_{S}-[\mathrm{F} 770 \mathrm{~W}],[\mathrm{F} 770 \mathrm{~W}]\right)$ plane.

In the $([\mathrm{F} 1000 \mathrm{~W}]-[\mathrm{F} 1500 \mathrm{~W}],[\mathrm{F} 1000 \mathrm{~W}])$ and $([\mathrm{F} 1000 \mathrm{~W}]-$ $[\mathrm{F} 2100 \mathrm{~W}],[\mathrm{F} 1000 \mathrm{~W}])$ planes the dual chemistry stars populate the same region where we find the sources with low obscuration.

\section{M-TYPE EVOLVED STARS IN THE COLOUR-COLOUR DIAGRAMS}

The distribution of the several $M$ stars classes discussed in the previous section in the colour-colour diagrams can be used to identify the different obscuration sequences and to select those planes where the various groups can be distinguished more clearly. In this case we lack significant information on the luminosity of the individual sources, but we gain some more data on the optical depth of the circumstellar envelopes. We show here the population of the stars in the sample used in the present investigation on the ([F770W]-[F2550W], $\left.K_{S^{-}}[\mathrm{F} 770 \mathrm{~W}]\right)$, $\left(K_{S^{-}}[\mathrm{F} 770 \mathrm{~W}], \quad[\mathrm{F} 1500 \mathrm{~W}]-[\mathrm{F} 1800 \mathrm{~W}]\right), \quad([\mathrm{F} 1000 \mathrm{~W}]-[12.5]$, $[\mathrm{F} 1500 \mathrm{~W}]-[\mathrm{F} 1800 \mathrm{~W}]), \quad([\mathrm{F} 770 \mathrm{~W}]-[\mathrm{F} 1000 \mathrm{~W}], \quad[\mathrm{F} 1500 \mathrm{~W}]-$ $[\mathrm{F} 2550 \mathrm{~W}])$ planes. We base the discussion on the results commented in the previous section.

As shown in the top, left panel of Fig.11, in the ([F770W]-[F2550W], $\left.K_{S^{-}}[\mathrm{F} 770 \mathrm{~W}]\right)$ plane, low-mass stars (magenta triangles; Sect. 4.2), and massive AGBs (green squares; Sect. 4.3), follow similar obscuration patterns, the latter extending to redder colours, because they reach higher optical depths. The two locii overlap in this case, because the main factor affecting the position of the stars is the optical depth; this allows us to draw a unique obscuration line. The trend is approximately horizontal up to $\tau_{10} \sim 0.1$, $[\mathrm{F} 770 \mathrm{~W}]-[\mathrm{F} 2550 \mathrm{~W}] \sim 2$; this behaviour is consistent with the arguments presented in Sect. 4.2, related to the modification of the shape of the SED, when the main silicate feature appears. For higher $\tau_{10}$, as shown in the right panel of Fig. 2, the SED is shifted to the mid-IR, which diminishes the flux in the near-IR, thus triggering a significant reddening of the $K_{S^{-}}[\mathrm{F} 770 \mathrm{~W}]$ colours: the sequences turn upwards in the diagram.

The stars with iron dust, both AGB and post-AGB, and those with dual-dust chemistry, discussed respectively in Sect. 4.4 and 4.5, are located in a diagonal sequence above the two afore mentioned obscuration sequences. This is once more due to the particular morphology of their SED, which does not exhibit a clear minimum in the $8 \mu \mathrm{m}$ region.

The link between the position of the individual sources and the degree of obscuration of the circumstellar enve- lope is clear also in the $\left(K_{S}\right.$-[F770W], [F1500W]-[F1800W]) plane, shown in the top, right panel of Fig.11. Low-mass and massive AGB stars describe obscuration sequences that extend to the red, with $K_{S^{-}}[\mathrm{F} 770 \mathrm{~W}]$ and [F1500W]-[F1800W] spanning a range of $\sim 6 \mathrm{mag}$ and $\sim 1 \mathrm{mag}$, respectively. Among the colours considered so far, [F1500W]-[F1800W] is the least sensitive to $\tau_{10}$, because, as shown in Fig. 2, the presence of the secondary silicate feature at $18 \mu \mathrm{m}$ is accompanied by a general increase in the stellar flux in all the spectral region between 10 and $25 \mu \mathrm{m}$ : both the [F1500W] and $[\mathrm{F} 1800 \mathrm{~W}]$ fluxes increase when a prominent secondary silicate feature is present in the spectrum. This is the reason why [F1500W]-[F1800W] never exceeds the maximum value, [F1500W]-[F1800W] 0.8, reached for $K_{S^{-}}[\mathrm{F} 770 \mathrm{~W}] \sim 2$.

The peculiar shape of the SED in the $8 \mu \mathrm{m}$ region makes iron dust and dual dust chemistry stars populate regions of the plane below the main obscuration pattern. In particular, AGB stars surrounded by iron dust are clearly separated from post-AGB stars and low-luminosity objects, with $K_{S^{-}}[\mathrm{F} 770 \mathrm{~W}] \sim 1$, [F1500W]-[F1800W] 0.3. This is due to the steep rise of the SED at $\lambda \leqslant 8 \mu m$ of the iron dust AGB sources respect to their post-AGB counterparts and stars with dual-dust chemistry. An additional colour where iron post-AGB stars are clearly isolated is the [F1280W]-[F2550W]. This is due to the absence of the silicates secondary feature. This colour in combination with $K_{S^{-}}[\mathrm{F} 770 \mathrm{~W}]$, separates this group of sources from the obscuration sequence traced by the rest of the sample.

The bottom panels of Fig.11 show the distribution of the stars in the sample considered here in two additional planes proposed by Jones et al. (2017) to discriminate AGB stars from other sources, e.g. young stellar objects and planetary nebulae. ([F1000W]-[F1280W],[F1500W]-[F1800W]) is also useful to separate O-rich from C-rich AGB stars, while ([F770W]-[F1000W],[F1500W]-[F2550W]) is effective in distinguishing between galactic and non-galactic sources. Nevertheless, unlike the upper panels diagrams, the trend of the colours of the O-rich stars with the optical depth is not straightforward. This is because the $[\mathrm{F} 1000 \mathrm{~W}]$ and [F1280W] flux depend on the particular way with which the shape of the main silicate feature changes with $\tau_{10}$.

In the ([F1000W]-[F1280W],[F1500W]-[F1800W]) plane the position of the individual stars does not allow a clear identification of the obscuration properties. The stars with iron dust and their counterparts with dual dust chemistry can be distinguished more clearly: indeed their [F1500W][F1800W] colours are bluer for a given [F1000W]-[F1280W], because the depression of the flux at $15 \mu \mathrm{m}$ is lower in comparison to the stars whose main dust component is made up of silicates.

In the ([F770W]-[F1000W],[F1500W]-[F2550W]) plane the different classes of stars presented in the previous section partly overlap and the separation among the various groups is not straightforward.

\section{SUMMARY AND CONCLUSIONS}

A summary of the overall interpretation of the LMC sources published by Jones et al. (2012) is reported in Table 2. For each source the luminosity, coordinates, periods (when available), optical depth at $10 \mu \mathrm{m}$, dust mineralogy and evolu- 

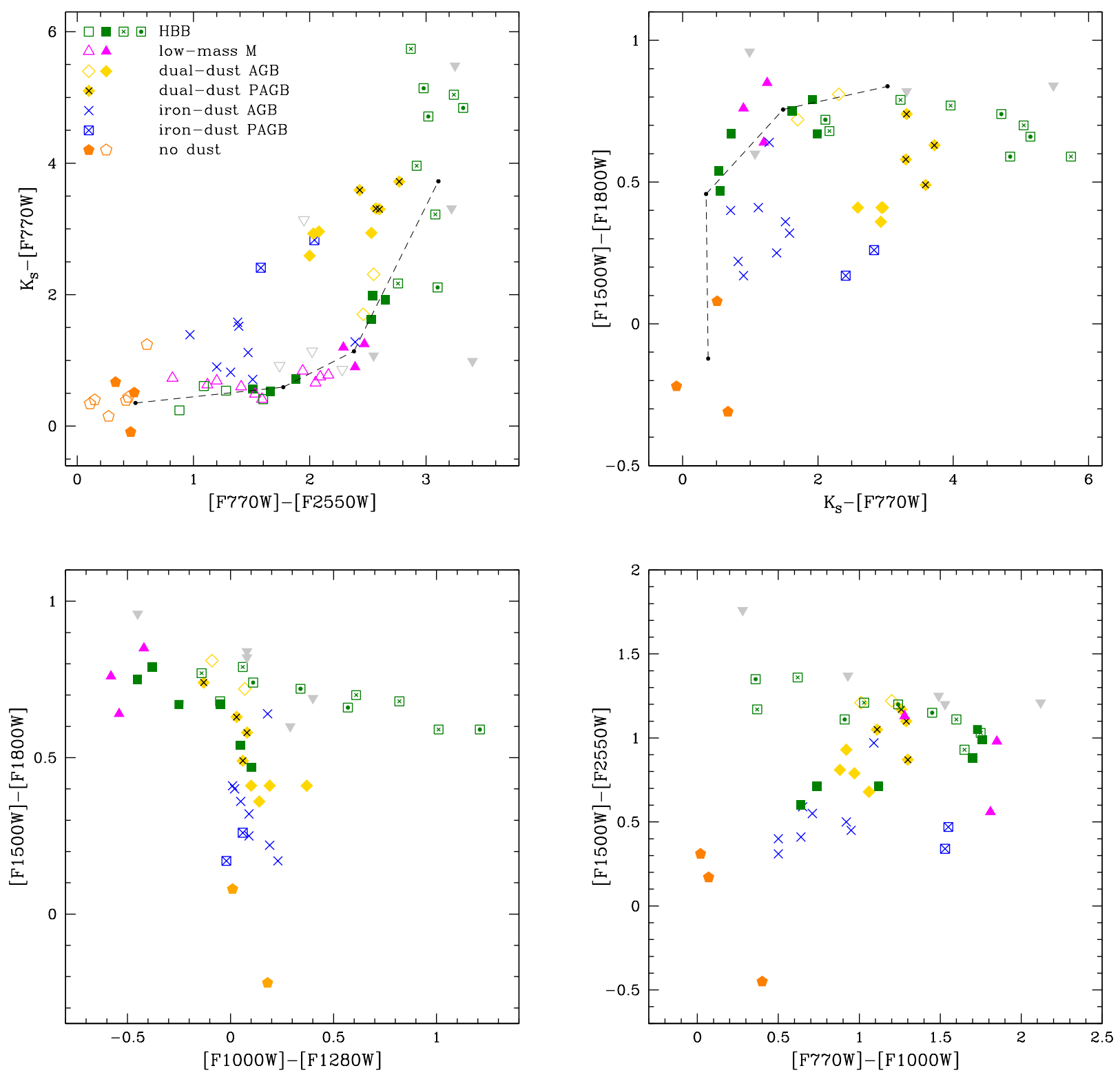

Figure 11. The evolved, M-type stars in the sample published by Jones et al. $(2012,2014,2017)$ in the various colour-colour planes, obtained by using the IR filters of the JWST.

tionary characterization are given. In the case of stars with dual-dust chemistry, we also report the optical depth and the percentages of solid carbon and $\mathrm{SiC}$ of the inner layer.

The table also includes the interpretation by GS18. We notice an overall satisfactory agreement with the latter exploration, but a few sources, for which the luminosities derived in the present study and in GS18 are substantially different. For what attains the sources SSID 121 and SSID 4007, the explanation is in the choice of GS18 to scale the IRS fluxes to match the IRAC and MIPS data. If we take this into account, the results are consistent. In the other few cases where the results are not consistent (i.e. SSID 38, 165, 4038 and 4483), we notice that the best fit obtained by
GS18 are indeed of poor quality, as witnessed by the high $\chi^{2}$ obtained, above 750 .

The results presented and discussed in the previous sections show that the LMC sample of the sources classified as evolved M-type AGB stars is mainly composed by two groups: a) low-mass stars, evolving through advanced AGB phases; b) massive AGB stars experiencing HBB. Additional stars included in the sample are low-metallicity massive AGBs surrounded by mostly iron dust, post-AGB stars and objects with dual-dust chemistry composition.

Among the two classes of objects listed in points a) and b), massive AGB stars are on the average more obscured 
than low-mass objects; however, the optical depths of the two groups partly overlap.

Whatever is the combination of JWST fiters adopted to build the observational planes, we expect that the separation between the two groups listed in points a) and b) can be more appreciated in the colour-magnitude diagrams compared to the colour-colour ones. Therefore, on general grounds, the former are a better diagnostic for the characterization of the individual sources.

The majority of these stars, with the exception of a few cases discussed in Sect. 4.4.2, are characterized by the presence of two silicate features, affecting the spectral range between $\sim 8$ and $\sim 22 \mu \mathrm{m}$. The shape of the SED in this wavelength interval depends on the optical depth, in turn related to the presence of dust in the circumstellar envelope of the star. As discussed in Sect. 2.3, the modality with which the SED changes with $\tau_{10}$ is not straightforward. This behaviour makes the filters falling within the afore mentioned spectral range to be of little help in the interpretation of the observations, because the obscuration patterns traced in the observational planes built with these filters present turning points and deviations, which prevents a straight understanding of the results (see Figs. 4 and 11).

As discussed in previous sections, a key-quantity in the interpretations of dusty M-stars is the [F770W] flux. This is because, as far as the dust around the stars is dominated by the silicate component, the [F770W] flux is less sensitive to the details of the changes in the shape of the SED with the degree of obscuration, compared to the filters in the $8 \mu \mathrm{m}<\lambda<22 \mu \mathrm{m}$ spectral region. On the other hand, the [F770W] flux is extremely sensitive to the presence of iron dust or, more generally, of featureless dust species and can therefore be efficiently used to check whether the sample encompasses iron dust stars and dual-chemistry sources.

Still within the mid-IR domain, the [F2550W] flux proves an important tool for the characterization of the individual sources, as it is sensitive to the overall degree of obscuration in the circumstellar envelope.

Turning to the near-IR, the $K_{S}$ flux is also extremely sensitive to the optical depth, although the measurements of the flux in the near-IR spectral regions can be hard for the most extremely obscured sources.

Based on the arguments given above, we conclude that the color-magnitude ([F770W]-[F2550W], [F770W]) plane is the best diagnostic for dusty M-stars, allowing the identification of the following: a) two separated obscuration patterns, holding for massive stars undergoing $\mathrm{HBB}$ and for their lower mass counterparts; b) stars surrounded by iron dust, interpreted as the progeny of low-metallicity massive AGBs; c) post-AGB stars with a dual-chemistry dust composition.

The same two groups of obscured, M-stars can be also distinguished in the color-magnitude $\left(K_{S^{-}}[\mathrm{F} 770 \mathrm{~W}]\right.$, $[\mathrm{F} 770 \mathrm{~W}])$ plane. In this diagram the post-AGB stars are more clearly separated and the $K_{S}$ - $[\mathrm{F} 770 \mathrm{~W}]$ colour of massive AGBs is more sensitive than [F770W]-[F2550W] to the degree of obscuration. However, the obscuration pattern traced by low-mass dusty AGBs is extremely short and, as discussed previously, the estimate of the $K_{S}$ flux is hampered by the presence of large quantities of dust.

The latter set of filters can also be used to plot the stars in the colour-colour ([F770W]-[F2550W], $\left.K_{S^{-}}[\mathrm{F} 770 \mathrm{~W}]\right)$ plane. In this case a clear degree of obscuration is present, despite the sequences of massive AGBs and low-mass stars are not separated. Post-AGB stars, iron-dust sources and dual-dust chemistry stars can be distinguished, although they populate zones of the plane close to the main obscuration pattern.

Among the colour-colour planes, an alternative possibility to select the different groups of stars is $\left(\left[K_{S^{-}}[\mathrm{F} 770 \mathrm{~W}]\right.\right.$, [F1500W]-[F1800W]).

In summary, future $J W S T$ investigations aimed at the characterization of evolved $M$ stars in galaxies must consider as a priority the collection of data in the F770W and F2550W filters. The F210M filter will be also useful to build combinations of near-IR and mid-IR photometry to distinguish the different obscuration sequences and characterize the individual sources, although its use is limited by the extremely low near-IR fluxes of the most obscured stars.

\section{ACKNOWLEDGEMENTS}

FDA and DAGH acknowledge support from the State Research Agency (AEI) of the Spanish Ministry of Science, Innovation and Universities (MCIU) and the European Regional Development Fund (FEDER) under grant AYA201788254-P.

DK acknowledges the support of the Australian Research Council (ARC) Discovery Early Career Research Award (DECRA) grant (95213534).

MT acknowledges support the European Research Council (ERC) under the European Union's Horizon 2020 research innovation programme (Grant Agreement ERC-StG 2016, No 716082 'GALFOR', PI: Milone) and from MIUR through the the FARE project R164RM93XW 'SEMPLICE' (PI: Milone).

\section{REFERENCES}

Begemann B., Dorschner J., Henning T., Mutschke H., Gürtler J., Kömpe C., Nass R., 1997, ApJ, 476, 199

Blöcker T., Schönberner D., 1991, A\&A, 244, L43

Blum R. D., Mould J. R., Olsen K. A., et al. 2006, AJ, 132, 2034

Boothroyd A. I., Sackmann I.-J. 1988, ApJ, 328, 653

Bouchet P., García-Marín M., Lagage P.-O., et al. 2015, PASP, 127, 612

Bowen G. H., 1988, ApJ, 329, 299

Boyer M. L., McQuinn K. B. W., Barmby P., et al., 2015, ApJS, 216, 10

Cameron A. G. W., Fowler W. A. 1971, ApJ, 164, 111

Cherchneff I., 2006, A\&A, 456, 1001

Dell'Agli F., Ventura, P., García Hernández, D. A., et al. 2014a, MNRAS, 442, L38

Dell'Agli F., García-Hernández D. A., Rossi C., et al. 2014b, MNRAS, 441, 1115

Dell'Agli F., Ventura P., Schneider, R., et al. 2015a, MNRAS, 447, 2992

Dell'Agli F., García-Hernández D. A., Ventura P., et al. 2015b, MNRAS, 454, 4235

Dell'Agli F., Di Criscienzo M., Boyer M. L., GarcíaHernández, D. A. 2016, MNRAS, 460, 4230 
$M$-stars in the $L M C$

Table 2. The summary of the overall interpretation of the LMC sources analyzed in this work, including the interpretation by GS18. This table is available in its entirety in machine-readable form.

\begin{tabular}{|c|c|c|c|c|c|c|c|c|}
\hline SSID & RA DEC & Period (d) & $\mathrm{L} / \mathrm{L}_{\odot}$ & $\%\left(\mathrm{Sil}, \mathrm{Al}_{2} \mathrm{O}_{3}, \mathrm{Fe}\right)$ & $\tau_{10}$ & Type & $\mathrm{L} / \mathrm{L}_{\odot}(\mathrm{GS} 18)$ & $\%\left(\mathrm{Sil}, \mathrm{Al}_{2} \mathrm{O}_{3}, \mathrm{Fe}\right)(\mathrm{GS} 18)$ \\
\hline 1 & $69.338-70.579$ & - & 7000 & $(60,40,0)$ & $4.5 \times 10^{-3}$ & Low-mass M & 5894 & $(40,60,30)$ \\
\hline 6 & $72.393-69.097$ & - & 18000 & $(15,0,85)$ & $2 \times 10^{-3}$ & Iron-dust PAGB & - & - \\
\hline 8 & $72.869-69.930$ & 884 & 2100 & $\begin{array}{c}(60,10,30) \\
\text { C } 95, \mathrm{SiC} 5\end{array}$ & $\begin{array}{l}3.2 \times 10^{-2} \\
1.5 \times 10^{-2}\end{array}$ & Dual-Dust AGB & 1660 & $(70,30,30)$ \\
\hline 13 & $73.290-68.286$ & 916 & 5500 & - & - & No dust & 5545 & $(40,60,1)$ \\
\hline 22 & $74.097-69.463$ & - & 4700 & $\begin{array}{c}(60,5,35) \\
\text { C 95, SiC } 5\end{array}$ & $\begin{array}{c}1.5 \times 10^{-2} \\
8 \times 10^{-3}\end{array}$ & Dual-dust AGB & 7524 & $(70,30,30)$ \\
\hline 38 & $76.120-67.690$ & 577 & 34000 & $(65,5,30)$ & $6.7 \times 10^{-2}$ & $\mathrm{HBB}$ & 14409 & $(100,0,30)$ \\
\hline 54 & $76.997-68.657$ & - & 4300 & $\begin{array}{c}(55,30,15) \\
\text { C } 100\end{array}$ & $\begin{array}{c}1.8 \times 10^{-2} \\
6 \times 10^{-3}\end{array}$ & Dual-dust AGB & 3257 & $(70,30,30)$ \\
\hline
\end{tabular}

Dell'Agli F., García-Hernández D. A., Schneider, R., et al. 2017, MNRAS, 467, 4431

Dell'Agli F., García-Hernández D. A., Ventura P., et al. 2018a, MNRAS, 475, 3098

Dell'Agli F., Di Criscienzo M., Ventura P., et al. 2018b, MNRAS, 479, 5035

Dell'Agli F., Di Criscienzo M., García-Hernández D. A., et al. 2019, MNRAS, 482, 4733

Di Criscienzo M., Dell'Agli F., Ventura P., et al. 2013, MNRAS, 433, 313

Feast M., 1999, PASP, 111, 775

Ferrarotti A. S., Gail, H.-P. 2002, A\&A, 382, 256

Ferrarotti A. S., Gail, H.-P. 2006, A\&A, 447, 553

Gail, H.-P. \& Sedlmayr E. 1999, A\&A, 347, 594

Gail H.-P., Wetzel S., Pucci A., Tamanai A. 2013, A\&A, 555, A119

Gielen C., Bouwman J., van Winckel H., et al. 2011, 533, A99

Gielen C., Cami J., Bouwman J., Peeters E., Min M., 2011, A\&A, 536, A54

Górny S. K., Perea-Calderón J. V., García-Hernández D. A., García-Lario P., Szczerba R., 2010, A\&A, 516, A39 Groenewegen M. A. T., Sloan G. C. 2018, A\&A, 609, A114

Hanner M., 1988, ioch.rept

Harris J., Zaritsky D. 2009, AJ, 138, 1243

Höfner S., Bladh S., Aringer B., Ahuja R., 2016, A\&A, 594, A108

Höfner S., Olofsson H., 2018, A\&ARv, 26, 1

Iben, I. Jr. 1974, ARA\&A, 12, 215

Javadi A., van Loon J. T., Khosroshahi H. 2016, Memorie della Societá Astronomica Italiana, 87, 278

Jones O. C., Kemper F., Sargent B. A., et al. 2012, MNRAS, 427, 3209

Jones O. C., Kemper F., Srinivasan S., McDonald I., Sloan G. C., Zijlstra, A. A. A. 2014, MNRAS, 440, 631

Jones O. C., Meixner M., Justtanont K., Glasse, A. 2017, ApJ, 841, 15

Kamath D., Wood P. R., Van Winckel H., 2014, MNRAS, 439, 2211

Kamath D., Wood P. R., Van Winckel H., 2015, MNRAS, 454, 1468

Karakas A. I. 2010, MNRAS, 403, 1413

Karakas A. I., Lattanzio, J. C. 2014, PASA, 31, e030

Karakas A. I., Lugaro M., Carlos M., Cseh B., Kamath D., García-Hernández D. A. 2018, MNRAS, 477, 421

Kemper F., Woods P. M., Antoniou V., et al. 2010, PASP,
122,683

Li Y., Bryan G. L., Quataert E. 2019, arXiv:1901.10481

Marigo P., Girardi L., Bressan A. 1999, A\&A, 344, 123

Marigo P. 2002, A\&A, 387, 507

Marini E., Dell'Agli F., Di Criscienzo M., Puccetti S., García-Hernández D. A., Mattsson L., Ventura P. 2019a, ApJL, 871, L16

Marini E., Dell'Agli F., García-Hernández D. A., Groenewegen M. A. T., Puccetti S., Ventura P., Villaver E., 2019b, MNRAS, 488, L85

Matsuura M., et al., 2014, MNRAS, 439, 1472

Mazzitelli I., D'Antona F., Ventura P. 1999, A\&A, 348, 846

Meixner M. et al., 2006, AJ, 132, 2268

Miller Bertolami M. M. 2016, A\&A, 588, A25

Nanni A., Bressan A., Marigo P., Girardi L., 2013, MNRAS, 434, 2390

Nanni A. Bressan A. Marigo P. Girardi L., 2014, MNRAS, 438, 2328

Nanni A., Groenewegen M. A. T., Aringer B., et al. 2019, MNRAS, 487, 502

Nenkova M., Ivezic Z., Elitzur M., 1999, LPICo, 20, LPICo.969

Ordal M. A., Bell R. J., Alexander R. W., et al. 1988, Applied Optics, 27, 1203

Ossenkopf V., Henning T., Mathis J. S., 1992, A\&A, 261, 567

Pegourie B., 1988, A\&A, 194, 335

Perea-Calderón J. V., García-Hernández D. A., GarcíaLario P., Szczerba R., Bobrowsky M. 2009, A\&A, 495, L5

Renzini A., Voli M. 1981, A\&A, 94, 175

Riebel D., Meixner M., Fraser O., Srinivasan S., Cook K., Vijh U. 2010, ApJ, 723, 1195

Riebel D., Srinivasan S., Sargent B., Meixner M., 2012, ApJ, 753, 71

Rieke G. H., Wright G. S., Böker T., et al. 2015, PASP, 127,584

Schlegel D. J., Finkbeiner D. P., Davis M., 1998, ApJ, 500, 525

Schneider R., Valiante R., Ventura P., et al. 2014, MNRAS, 442, 1440

Sharp C. M., Huebner W. F. 1990, ApJS, 72, 417

Skrutskie M. F., et al., 2006, AJ, 131, 1163

Soszyński I., Udalski A., Szymański M. K., et al. 2009, AcA, 59, 239

Srinivasan S. et al., 2009, AJ, 137, 4810 
Srinivasan S., Sargent B. A., Matsuura M., et al. 2010, A\&A, 524, A49

Srinivasan S., Sargent B. A., Meixner M. 2011, A\&A, 532, A54

Trabucchi M., Wood P. R., Montalbán J., et al. 2018, ASPC, 111, ASPC..514

van Aarle E., van Winckel H., Lloyd Evans T., Ueta T., Wood P. R., Ginsburg A. G., 2011, A\&A, 530, A90

van Winckel H., 2003, ARA\&A, 41, 391

Vassiliadis E., Wood P. R. 1993, ApJ, 413, 641

Ventura P., D'Antona F. 2005a, A\&A, 431, 279

Ventura P., D'Antona F. 2005b, A\&A, 439, 1075

Ventura P., Marigo P. 2009, MNRAS, 399, L54

Ventura P., Marigo P. 2010, MNRAS, 408, 2476

Ventura P., Di Criscienzo M., Schneider R., et al. 2012, MNRAS, 420, 1442

Ventura P., Di Criscienzo M., Carini R., D'Antona F. 2013, MNRAS, 431, 3642

Ventura P., Dell'Agli F., Schneider R., et al. 2014, MNRAS, 439, 977

Ventura P., García-Hernández D. A., Dell'Agli F., et al. 2016, ApJL, 831, L17

Ventura P., Karakas A., Dell'Agli F., García-Hernández D. A., Guzman-Ramirez, L. 2018, MNRAS, 475, 2282

Wachter A., Schröder K. P., Winters J. M., Arndt T. U., Sedlmayr E., 2002, A\&A, 384, 452

Wachter A., Winters J. M., Schröder K. P., Sedlmayr E., 2008, A\&A, 486, 497

Waters L. B. F. M., et al., 1998, Natur, 391, 868

Woitke P., Helling C., Winters J. M., Jeong K. S. 1999, A\&A, 348, L17

Woods P. M. Oliveira J. M., Kemper F., et al. 2011, MNRAS, 411, 1597

Zaritsky D., Harris J., Thompson I. B., \& Grebel E. K. 2004, AJ, 128, 1606

Zhukovska S., Henning T. 2013, A\&A, 555, A99

This paper has been typeset from a $\mathrm{T}_{\mathrm{E}} \mathrm{X} / \mathrm{LAT}_{\mathrm{E}} \mathrm{X}$ file prepared by the author. 